\title{
Development of Control System Functional Capabilities within the IES Plug-and-Play Simulation Environment
}

IES Simulation Ecosystem Control System Development

Nuclear Science and Engineering Division 
About Argonne National Laboratory

Argonne is a U.S. Department of Energy laboratory managed by UChicago Argonne, LLC under contract DE-AC02-

$06 \mathrm{CH} 11357$. The Laboratory's main facility is outside Chicago, at 9700 South Cass Avenue, Argonne, Illinois 60439. For information about Argonne and its pioneering science and technology programs, see www.anl.gov.

\section{DOCUMENT AVAILABILITY}

Online Access: U.S. Department of Energy (DOE) reports produced after 1991 and a growing number of pre-1991 documents are available free at OSTI.GOV (http://www.osti.gov/), a service of the US Dept. of Energy's Office of Scientific and Technical Information.

Reports not in digital format may be purchased by the public from the

National Technical Information Service (NTIS):

U.S. Department of Commerce

National Technical Information Service

5301 Shawnee Rd

Alexandria, VA 22312

www.ntis.gov

Phone: (800) 553-NTIS (6847) or (703) 605-6000

Fax: (703) 605-6900

Email: orders@ntis.gov

Reports not in digital format are available to DOE and DOE contractors from the Office of Scientific and Technical Information (OSTI):

U.S. Department of Energy

Office of Scientific and Technical Information

P.O. Box 62

Oak Ridge, TN 37831-0062

www.osti.gov

Phone: (865) 576-8401

Fax: (865) 576-5728

Email: reports@osti.gov

\section{Disclaimer}

This report was prepared as an account of work sponsored by an agency of the United States Government. Neither the United States Government nor any agency thereof, nor UChicago Argonne, LLC, nor any of their employees or officers, makes any warranty, express or implied, or assumes any legal liability or responsibility for the accuracy, completeness, or usefulness of any information, apparatus, product, or process disclosed, or represents that its use would not infringe privately owned rights. Reference herein to any specific commercial product, process, or service by trade name, trademark, manufacturer, or otherwise, does not necessarily constitute or imply its endorsement, recommendation, or favoring by the United States Government or any agency thereof. The views and opinions of document authors expressed herein do not necessarily state or reflect those of the United States Government or any agency thereof, Argonne National Laboratory, or UChicago Argonne, LLC. 
ANL/NSE-20/35

\section{Development of Control System Functional Capabilities within the IES Plug-and-Play Simulation Environment}

IES Simulation Ecosystem Control System Development

prepared by

Haoyu Wang, Roberto Ponciroli, Richard B. Vilim

Nuclear Science and Engineering Division, Argonne National Laboratory

and

Andrea Alfonsi

Reactor Systems Design \& Analysis Division, Idaho National Laboratory

September 30, 2020 
Development of Control System Functional Capabilities within the IES Plug-and-Play Simulation Environment

September 30, 2020 


\section{Table of Contents}

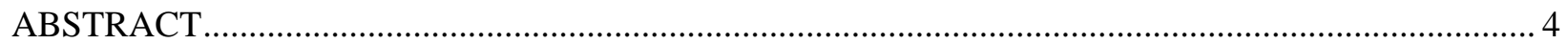

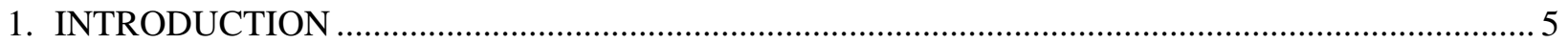

1.1. Traditional solutions to account for Implicit constraints .......................................................... 5

1.2. Proposed solution to account for Implicit constraints: Reference Governor-based scheme............ 7

2. DEFINITION OF THE RG-BASED FEEDBACK SCHEME ....................................................... 7

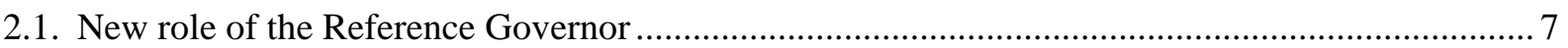

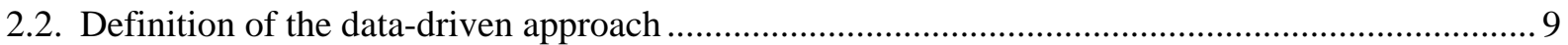

2.3. Definition of the LTI model-based workflow........................................................................... 10

2.4. Definition of the LTV model-based workflow …........................................................................ 11

3. DEFINITION OF A STATE VARIABLES SELECTION ALGORITHM ......................................... 13

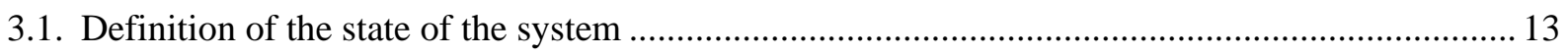

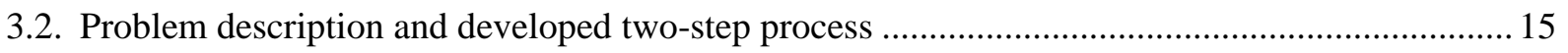

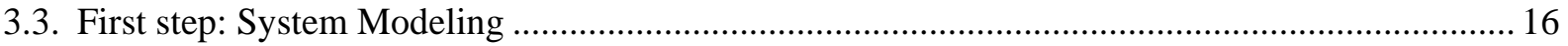

3.4. Second step: Principal Component Analysis .............................................................................. 16

3.5. Third step: Recursive Feature Elimination ….............................................................................. 18

4. DEFINITION OF THE SYSTEM IDENTIFICATION ALGORITHM .............................................. 18

5. DEFINITION OF THE REFERENCE GOVERNOR ALGORITHM .............................................. 21

6. VALIDATION OF THE DEVELOPED OPTIMIZATION SCHEME ............................................24

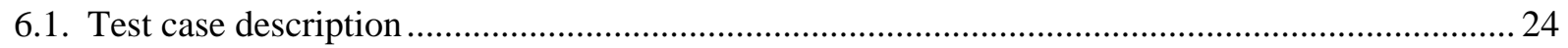

6.2. Implementation in RAVEN simulation environment: simulation outcomes ................................ 25

7. DEVELOPMENT OF A CONTROL SYSTEMS MODELICA LIBRARY .......................................26

7.1. Definition of the "Plug-and-Play" concept for control-oriented modeling.................................... 26

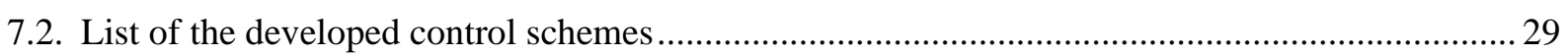

7.3. Testing of the Plug-and-play configuration on SES model ….................................................... 30

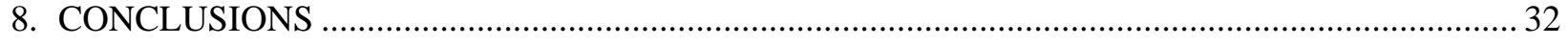

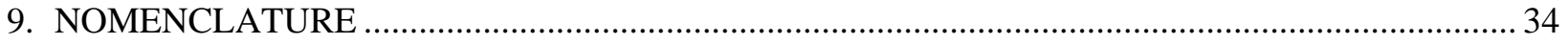

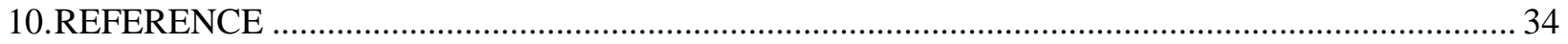




\section{List of Figures}

Figure 1-1. Saturation effects-based solution to account for both explicit and implicit constraints.

Figure 1-2. Extended power dispatch optimization scheme. Both explicit and implicit constraints are accounted for by the power dispatcher.

Figure 1-3. Proposed two-step constrained optimization scheme. The power dispatcher takes care of the explicit constraints and the RG of the implicit constraints.

Figure 2-1. Originally proposed RG-based architecture for the RAVEN/Dymola coupling....................... 8

Figure 2-2. LTI model-based optimization scheme (state-space representation matrices are derived off-

line).

Figure 2-3. Graphical representation of the "rolling window" concept................................................... 12

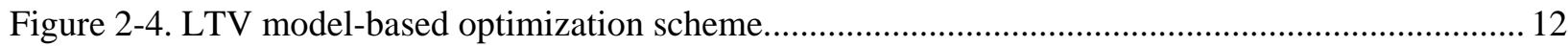

Figure 2-5. Configuration of the LTV model-based optimization scheme during the first iteration (on the

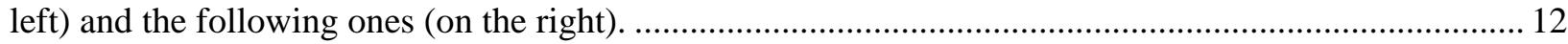

Figure 2-6. LTI model-based optimization scheme (state-space representation matrices are derived off-

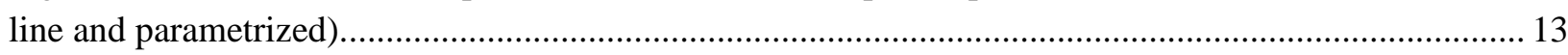

Figure 3-1. Dynamic system configuration. Input, Output and State variable are represented. ................. 14

Figure 3-2. State selection process workflow in RAVEN. ................................................................. 16

Figure 7-1. Dymola graphical interface of the SES model component (left), detailed view (right)........... 27

Figure 7-2. Graphical representation of a nuclear power plant operation by adopting a MIMO control

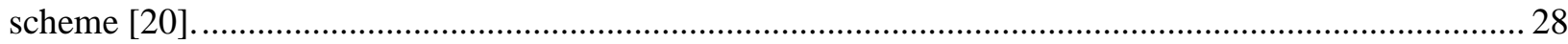

Figure 7-3. Dymola graphical interface of the BOP model component.................................................. 29

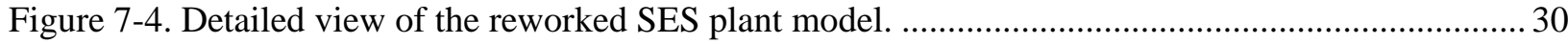

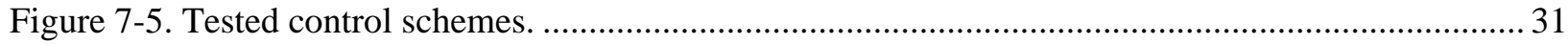

Figure 7-6. Simulation outcomes of the PID-based control scheme.................................................... 31

Figure 7-7. Simulation outcomes of the RG-Kalman-based control scheme............................................. 32 


\section{List of Tables}

Table 6-1. Selected components for the developed test-case. The main constraints and the corresponding

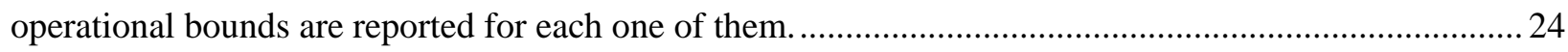

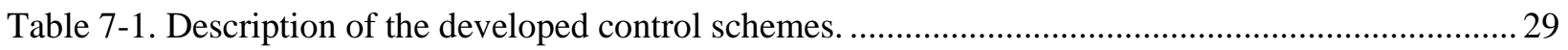




\section{ABSTRACT}

The concept of an Integrated Energy System (IES) is meant to combine different energy technologies in synergistic ways to achieve a more secure and economical energy supply. The RAVEN-based HYBRID framework is used to find the optimal installed capacity and the optimal economical dispatch of each component of the IES. A new RAVEN (Risk Analysis Virtual ENvironment) plugin for grid and capacity optimization (HERON) has been developed for optimizing the production variables of the IES given the demand profile. Currently, only the limits that affect the production variables and their corresponding time rates of change are considered (explicit constraints). However, other variables are additionally subject to constraints, but the associated limits are not accounted for (implicit constraints). In particular, for the power dispatch problem, the optimization algorithm takes into account the limits on the electrical power output and the corresponding hourly power variations but does not consider other constraints on process variables whose response effects the service life of the IES.

This report describes a scheme that allows accounting for implicit constraints without increasing the size of the optimization problem. The Reference Governor (RG) algorithm is traditionally used for enforcing state and control constraints by modifying the set-point trajectories supplied to the feedback regulators. In our application, the RG is coupled within an iterative loop with the HERON-power dispatcher to generate optimal trajectories that ensure the operational constraints are met. A data-driven procedure to derive a representation of the dynamics of the controlled system was developed. First, the variables that represented the state of the system are selected (PCA-based approach), and then state-space representation matrices are derived from the collected measurements (DMDc algorithm). A preliminary version of the developed workflow based on Linear Time Invariant matrices was assessed by adopting a two-unit test case. More sophisticated versions of this workflow foreseeing the on-line derivation of system matrices will be deployed in FY 2021.

Finally, a "plug-and-play" library of controllers and state observers was developed in Dymola. Some aspects of the current configuration of the IES unit components, e.g., the encapsulation of the control schemes into dedicated blocks, are consistent with the "plug-and-play" philosophy. Other features, e.g., the system buses collecting the input and the output variables, are not. For this reason, once listed and described the limits of the current configuration, necessary modifications to the plant model interface are presented. As a test-case, the interfaces of the SES model in the RAVEN-based HYBRID framework were reworked accordingly, and two different control schemes were applied to the same plant model. 


\section{INTRODUCTION}

HERON (Holistic Energy Resource Optimization Network) [1], a newly-developed RAVEN (Risk Analysis Virtual ENvironment) plugin for grid and capacity optimization, allows optimizing the desired production variables of Integrated Energy System (IES), e.g., electrical power, thermal power, hydrogen mass flow rate, etc., given the demand profile. The currently implemented optimization algorithm only considers the explicit constraints, i.e., the limits that affect the instantaneous values of the production variables and the corresponding rate of variations. Similarly, there might be other variables that have constraints, but these limits are not accounted for (implicit constraints) [2]. To understand this aspect, let us consider the power dispatch problem for the IES system, i.e., the optimization of the electrical power output by each one of the IES units. At the moment, the optimization algorithm (power dispatcher) takes into account the limits on the electrical power output and the corresponding hourly power variations (ramp-rate limits). At the same time, when the operation of a generating unit is optimized, there are other constraints that need be considered.

- Electrical Power constraints (Explicit constraints): the electrical power output can assume any value within a characteristic window, i.e., $P_{\min } \leq P(t) \leq P_{\max }$ (power limits). At the same time, electrical power variations cannot be too steep, i.e., $\left|\frac{d P}{d t}\right| \leq\left(\frac{d P}{d t}\right)_{\max }$ (ramp-rate limits).

- Thermo-mechanical constraints (Implicit constraints): to avoid thermo-mechanical stress exceeding the design limits, temperature, pressure and flow rate need to be constrained as well, i.e., $T_{\min } \leq T(t) \leq T_{\max }, p_{\min } \leq p(t) \leq p_{\max }, w_{\min } \leq w(t) \leq w_{\max }$.

\subsection{Traditional solutions to account for Implicit constraints}

The respect of both explicit and implicit constraints is crucial to obtain power dispatches that will ensure meeting the demand without causing damages to the IES system. For each component, i.e., nuclear reactor, gas turbine, thermal energy storage, industrial process, etc., there are several temperatures, pressures and flow rates whose transients need to be bounded for the safe operation of the unit itself. If the operating conditions of the turbines, the compressor, the condenser etc. that constitute the IES system exceed the corresponding design limits, damages will be caused, the performance will degrade, and the availability will be affected. The goal is obtaining a solution that allows optimizing the cost function by maintaining the conditions of the IES system components within the normal operation range.

A widely diffused solution to prevent violating the constraints on the main process variables consists in setting saturations on the system actuators (Figure 1-1). The power dispatcher evaluates the optimal power set-points for the IES components by considering the explicit constraints, and issues these trajectories to the corresponding feedback controllers (i.e., PIDs). If suitable saturation effects are imposed, the performed control actions will be limited by upper and lower bounds. If properly calibrated, these bounds will prevent the main process variables from exceeding the 
corresponding design limits. Though effective in enforcing the respect of the implicit constraint, this approach is not viable. Because of the saturation effects, the final electrical power production might be lower than the expected one (the load demand might not be met). Similarly, overproduction scenarios might occur. In this way, the simulated power transients might be quite different from the optimal ones evaluated by the power dispatcher.

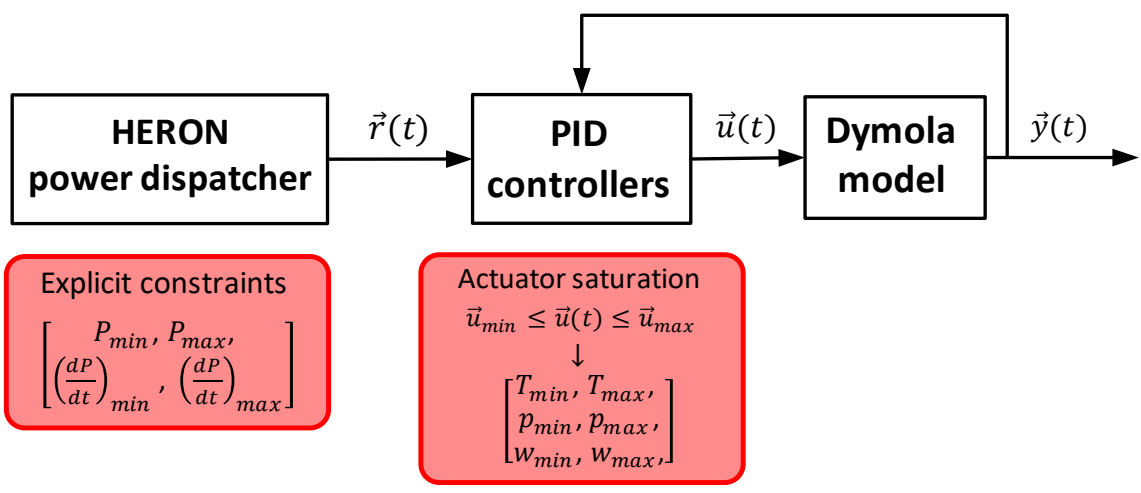

Figure 1-1. Saturation effects-based solution to account for both explicit and implicit constraints.

Another solution foresees the power dispatcher itself to account for the implicit constraints (Figure 1-2). In this case, specific transfer functions describing the effect of power set-point variations on the evolution of the constrained variables would be needed. This solution would allow obtaining optimal trajectories that could be issued to the system controllers without the issue of getting the system to explore off-design conditions. The main problems limiting the applicability of this approach are represented by (1) the effort needed to consistently implement these transfer function into the optimization scheme, and (2) the size of the ensuing optimization scheme (the response of a large number of variables should be simultaneously considered).

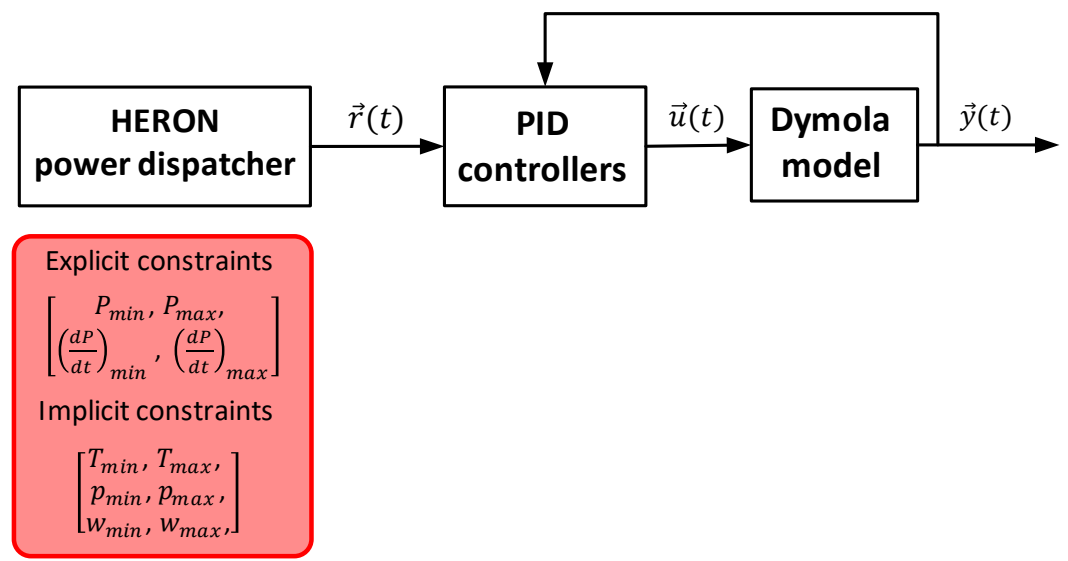

Figure 1-2. Extended power dispatch optimization scheme. Both explicit and implicit constraints are accounted for by the power dispatcher. 


\subsection{Proposed solution to account for Implicit constraints: Reference Governor-based scheme}

To handle the implicit constraints without increasing the complexity of the optimization problem, a Reference Governor (RG)-based algorithm was implemented into a dedicated RAVEN plugin and then coupled with the HERON power dispatcher (Figure 1-3). In the developed configuration, the power dispatch problem is solved in two-steps. First, HERON evaluates tentative power set-points $(\vec{r}(t))$ which meet the explicit constraints. These set-points are then issued to the RG. It is a sort of sanity check for the generated trajectories. If the HERON-evaluated set-points are compliant with the set of implicit constraints as well, then they will represent the optimal solution $(\vec{v}(t))$, and they can eventually be issued to the Dymola model; otherwise, the imposed set-points cannot be accepted since they will lead to the violation of some of the imposed constraints, and therefore they need to be re-evaluated. The RG is expected to assess the feasibility of the imposed operational transient at every time step (i.e., every hour or every five minutes depending on whether the hour-ahead or the real-time market is simulated). The advantage of this workflow is that the optimization problem can be simplified by de-coupling the set of constraints to be met, i.e., HERON will take care of optimizing the power set-points by accounting for the power limits (explicit constraints), the RG will assess that the issued set-points will not cause the violation of the temperature, pressure and flow rate limits (implicit constraints).

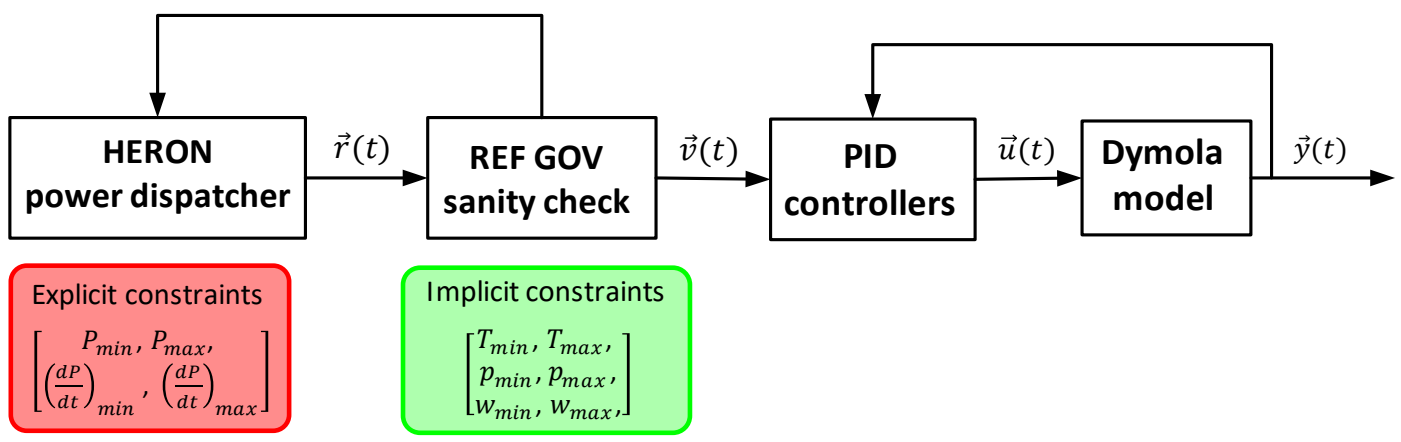

Figure 1-3. Proposed two-step constrained optimization scheme. The power dispatcher takes care of the explicit constraints and the RG of the implicit constraints.

\section{DEFINITION OF THE RG-BASED FEEDBACK SCHEME}

\subsection{New role of the Reference Governor}

The RG algorithm was conceived as an add-on scheme for enforcing pointwise-in-time state and control constraints by modifying the reference command to a well-designed (for small signals) closed-loop system [3][4][5]. The intent of the RG is to preserve, whenever possible, the response of the feedback loop system designed by conventional control techniques. This result is achieved by ensuring that the modified reference command is as close as possible to the original set-points subject to satisfy certain user-defined constraints, which might allow meeting limits on thermomechanical stresses, power ramps, etc. [5]. We assume that the PID controllers have already been designed to stabilize the system and provide good tracking properties in the absence of constraints, 
while the constraint fulfillment task is left to the RG. Commonly adopted controllers provide the system with asymptotic stability, acceptable dynamic performance, and robustness towards disturbances, but they cannot explicitly consider the presence of constraints on the operating condition of the component [5]. Unlike this approach which attempts to solve stabilization, tracking, and constraints fulfillment at the same time, the RG-based control scheme is based on a two-step approach [5]. First, primary compensated controllers are designed to stabilize the system and provide satisfactory tracking capabilities in the absence of constraints. Then, the RG block is added to enforce user-specified constraints.

Originally, the idea was to implement the RG into the Dymola simulation environment (Figure 2-1). First, the power set-points for the IES components were evaluated by the power dispatcher $(\vec{r}(t))$. Then, they were assessed by the RG. Adjusted set-points $(\vec{v}(t))$ were then generated and supplied to the PID controllers, according the instantaneous values of the state variables $(\vec{x}(t))$. The main limit of this approach is that it ensures that the constraints are met, but the final setpoints supplied to the Dymola model might not be optimal, i.e., the overall power output might be lower than the load demand. This is the same issue caused by the presence of saturations on the actuators. The initial set-points evaluated by the dispatcher are optimal but not feasible (i.e., they minimize the cost function and meet the demand by accounting for explicit constraints but not the implicit constraints), whereas the final set-points are sub-optimal but feasible (i.e., they meet both explicit and implicit constraints, but they do not meet the load demand).

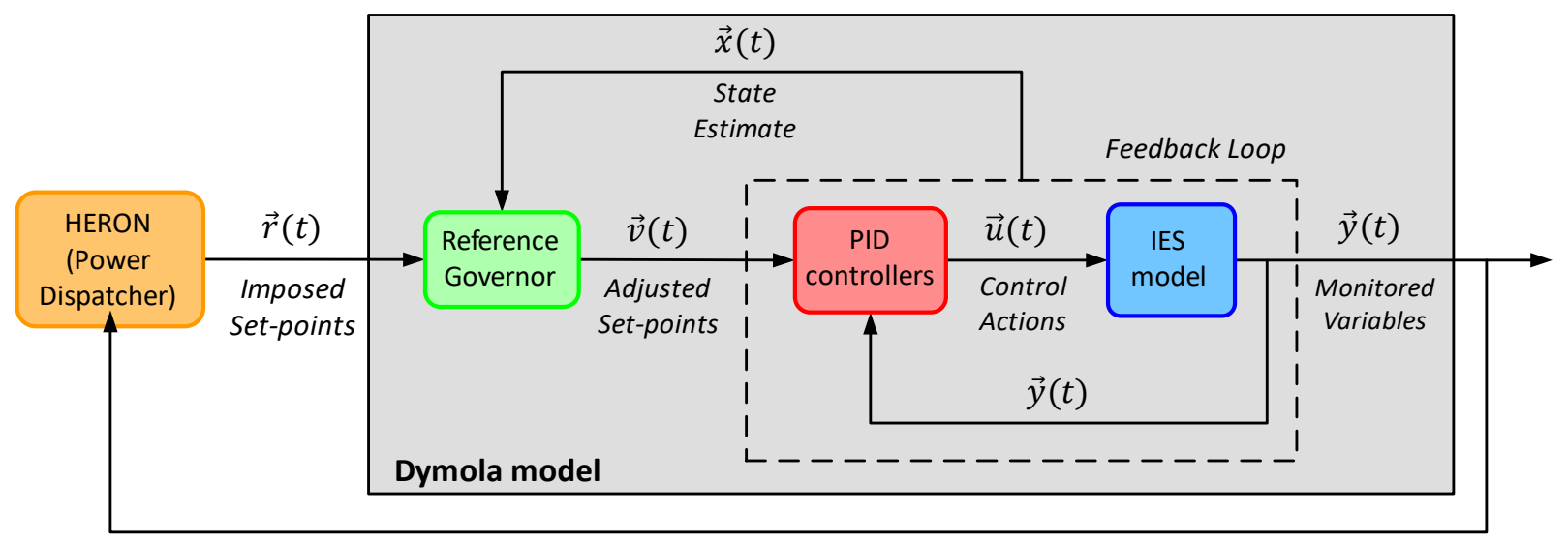

Figure 2-1. Originally proposed RG-based architecture for the RAVEN/Dymola coupling.

To address this problem, a different configuration was proposed. As shown in Figure 1-3, the RG is expected to iterate with the power dispatcher until an optimal and feasible solution is found. During every iteration, the power dispatcher will evaluate the optimal set-point trajectories over a certain time horizon (i.e., one day, one week, two weeks). The RG will assess that these values will not lead to the violation of the imposed constraints at any time step.

As for the integration of the RG with the power dispatcher, the RG returns the margins of the power set-point with respect to the limits imposed by the implicit constraints. When the system undergoes a certain power transient, constrained process variables (i.e., temperatures, pressures 
and flow rates) will evolve and their margins with respect to the corresponding upper and lower bounds will change. Given that the system input is represented by the power set-point, the margins on these temperatures, pressures, flow rates correspond to specific margins on the power set-point that can be evaluated by the RG algorithm. In particular, at every time-step, the RG will return the admissible range of values that could have been assumed by the power set-points before they would lead to the violation of any of the imposed constraints. This piece of information will be used by the power dispatcher during the second iteration of the power set-point optimization, and a more consistent power set-point trajectory will be evaluated.

\subsection{Definition of the data-driven approach}

To verify that the HERON-evaluated set-points are compliant with the set of implicit constraints, the RG algorithm needs a representation of the dynamics of the controlled system. Traditionally, a Linear Time Invariant (LTI) approximation is adopted. These models can be derived either (1) by linearizing the original high-fidelity simulator or (2) by adopting a system identification method.

- Linearization: the set of equations describing the system dynamics is linearized, either analytically or numerically, close to a reference equilibrium condition. This approach is rigorous, but it is not always feasible. In some applications, the presence of saturation effects or dead bands does not allow the linearization.

- System identifications methods: they are usually adopted when the studied system is too large or too complex to be linearized. First, a dynamic transient is simulated, and the input and the output variable responses are collected. Then suitable input/output data correlations are derived, and the LTI matrices are identified. This approach is robust, but the obtained matrices will represent a rough approximation if the system dynamics are nonlinear or if the unit operates over a wide range of operating conditions.

Theoretically, Dymola simulation environment allows performing the numerical linearization of a system of equations. Each of the IES components needs to be individually studied by specifying the corresponding input and output variables. Unfortunately, the currently implemented interfaces are not consistent with these requirements, and therefore this solution is not feasible. For the IES system, the system identification approach was then adopted. The proposed datadriven scheme foresees different steps, i.e., first the model to be implemented into the RG algorithm needs to be derived from the Dymola simulation outcomes, and then the RG algorithm can adjust the HERON-evaluated set-points. In Sections 2.3 and 2.4, the proposed workflows are described. In Sections 3, 4 and 5, the proposed algorithms to fulfil each of these steps are described. 


\subsection{Definition of the LTI model-based workflow}

In this section, the main steps of the LTI model-based workflow are identified and described. The corresponding configuration is represented in Figure 2-2.

- State Selection and System Identification: state-space representation matrices are derived off-line. Before the optimization process starts, power transients are simulated with the Dymola model of the IES to characterize the system dynamics over a wide range of operating conditions. Once the outcomes are collected, first the set of state variables is identified $(\hat{x}(t))$ and then the state-space representation matrices $(A, B, C, D)$ are derived. In this scheme, the simulation of power transients with Dymola, the selection of the state variables, and the derivation of the state-space representation matrices are performed off-line. During the optimization process, the same set of constant matrices is used to predict the system response ensuing from the imposed set-point variations.

- RG algorithm: at every time step, the RG evaluates the feasibility of the set-point. It receives as inputs (1) the tentative set-point trajectory from the Power Dispatcher $\left(\vec{P}^{j}\right)$, and (2) the LTI model matrices from the System Identification. It returns as outputs the margins of the set-point trajectory $\left(\delta \vec{P}_{\min }^{\max }\right.$ ) with respect to the imposed constraints (Section 2.1). These margins will be used to update the explicit constraints in the Power Dispatcher algorithm. Then, the setpoint trajectory will be re-calculated and re-submitted to the RG. Once the convergence is reached, the optimal set-point trajectory meeting both the explicit and the implicit constraints is returned $\left(\vec{P}^{\text {opt }}\right)$.

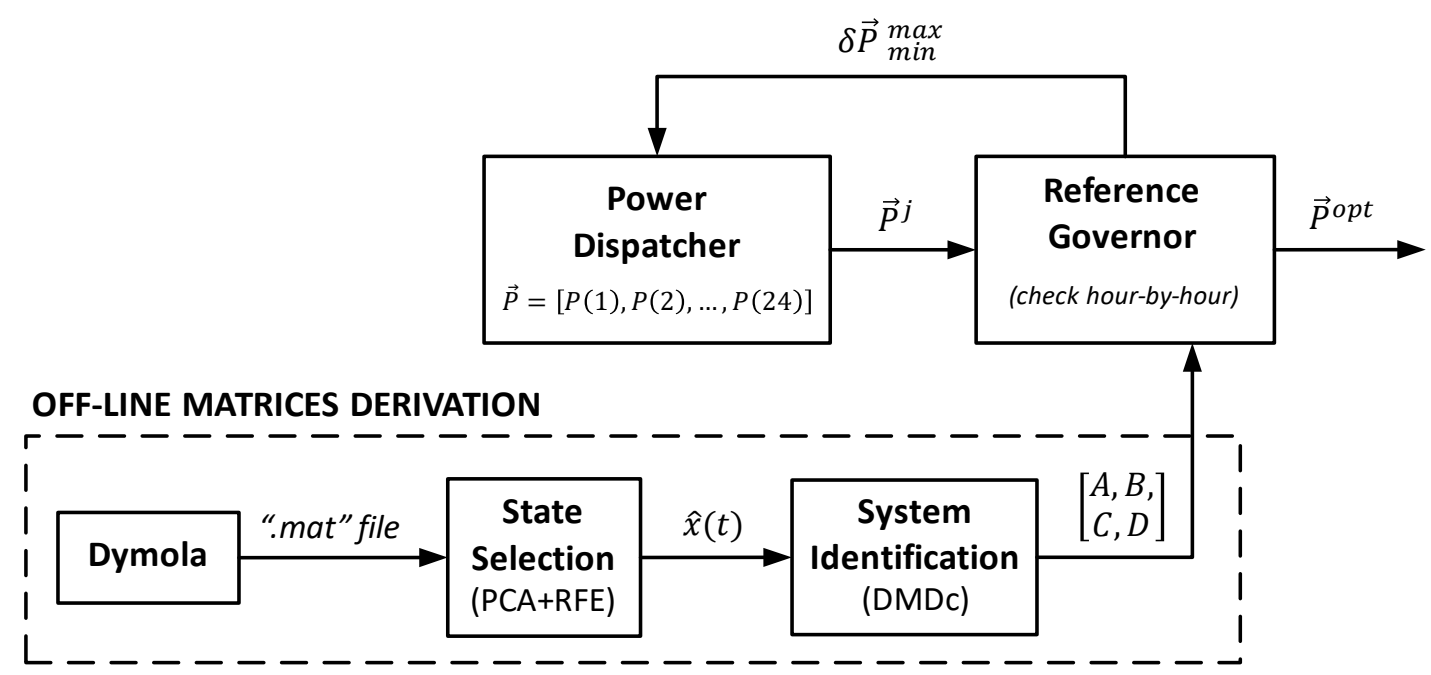

Figure 2-2. LTI model-based optimization scheme (state-space representation matrices are derived offline). 


\subsection{Definition of the LTV model-based workflow}

The scheme adopting constant matrices is efficient and ensures good convergence as long as the studied system is characterized by linear dynamics. On the other hand, if non-linear dynamic behavior is observed at certain operating conditions, the identified matrices will not accurately predict the evolution of the process variables, and the evaluated trajectories will be sub-optimal. With respect to the studied problem (IES unit components are expected to explore a wide range of operating conditions), an LTI model might not be accurate enough to represent the dynamics of the system. To this aim, a Linear Time Variant (LTV) model might be necessary. By continuously updating the matrices during the transient simulation, the most accurate approximation of the system dynamics will be obtained. In Figure 2-4, the graphical representation of the proposed solution is shown. With respect to the scheme represented in Figure 2-2, the Dymola model, the State Selection and the System Identification algorithms are continuously involved in the optimization process.

- State Selection: it takes as input the .mat file generated by Dymola simulation at every time step and identifies the variables that define the state of the system.

- System Identification: it collects the dynamic response of the state variables and the power set-point trajectories supplied to the Dymola model. Matrices are continuously updated during the transient simulation $(A(t), B(t), C(t), D(t))$. They represent the most accurate approximation of the system dynamics close to current operating conditions. The system identification method is coupled with the "rolling window" concept (Figure 2-3). A portion of the dynamic recent history of the studied physical system is tracked, the LTV representation of the system dynamics is derived, and the process variables evolution over the next few time steps is predicted. Then, the tracking window is moved, and the identification process is repeated.

- RG algorithm: as in the case of constant matrices optimization scheme, the RG evaluates the feasibility of the set-point trajectories supplied by the Power dispatcher. It receives as inputs (1) the tentative set-point trajectory from the Power Dispatcher $\left(\vec{P}^{j}\right)$, and (2) the LTV model matrices from the System Identification. At every time-step, the RG evaluates the power setpoint margins $\left(\delta \vec{P}_{\min }^{\max }\right)$ with respect to the imposed constraints, and it provides the Dymola model with the adjusted set-points $\left(\vec{P}^{o p t}\right)$ as long as constraints are not violated. The RG cannot supply Dymola with the dispatcher-provided set-point trajectory $\left(\vec{P}^{j}\right)$, otherwise the system will explore some forbidden operating condition and the RG algorithm will crash. To this aim, the instantaneous feasible power set-point is supplied to the Dymola model. 


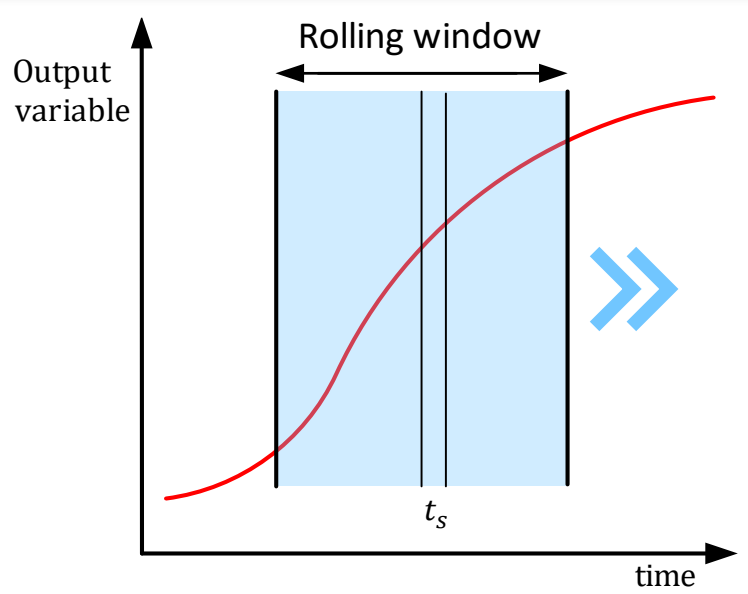

Figure 2-3. Graphical representation of the "rolling window" concept.

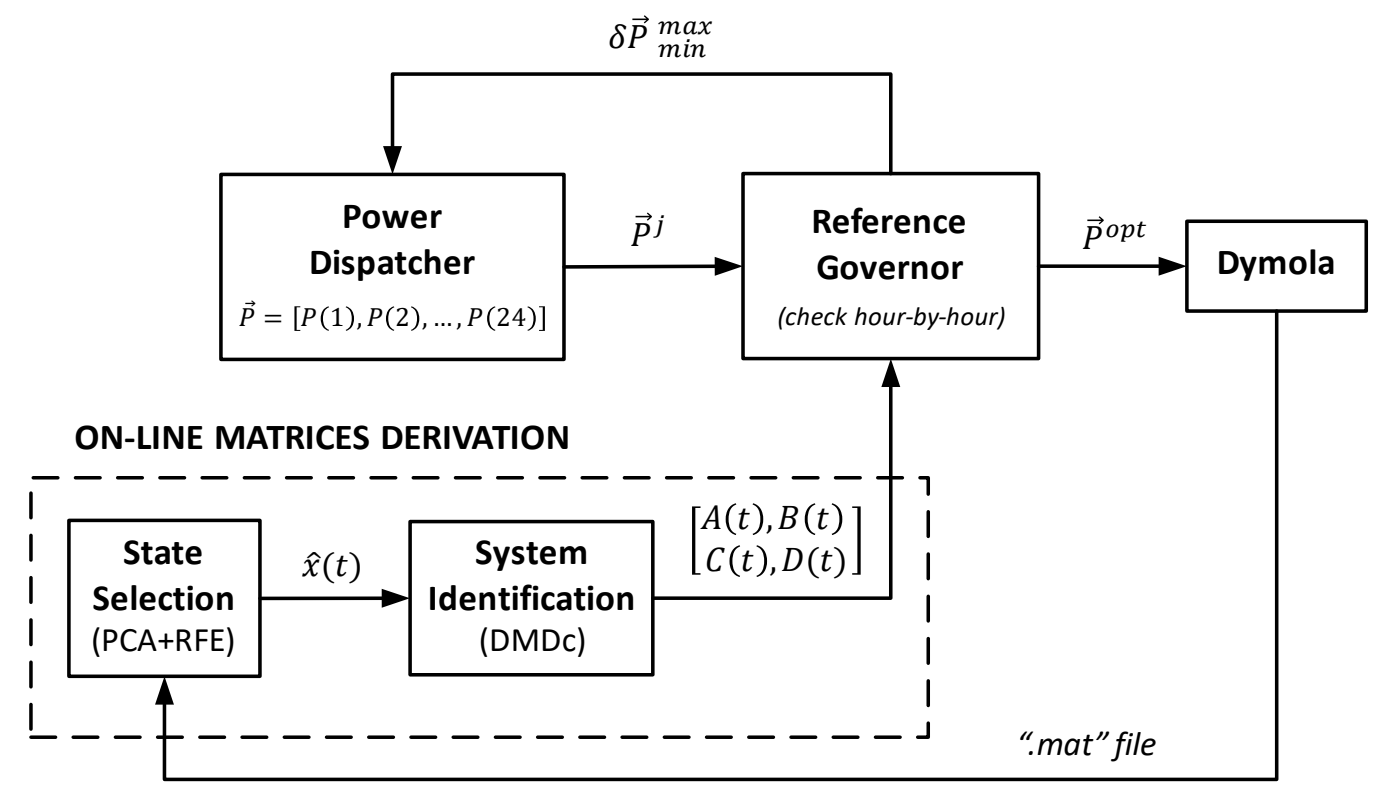

Figure 2-4. LTV model-based optimization scheme.

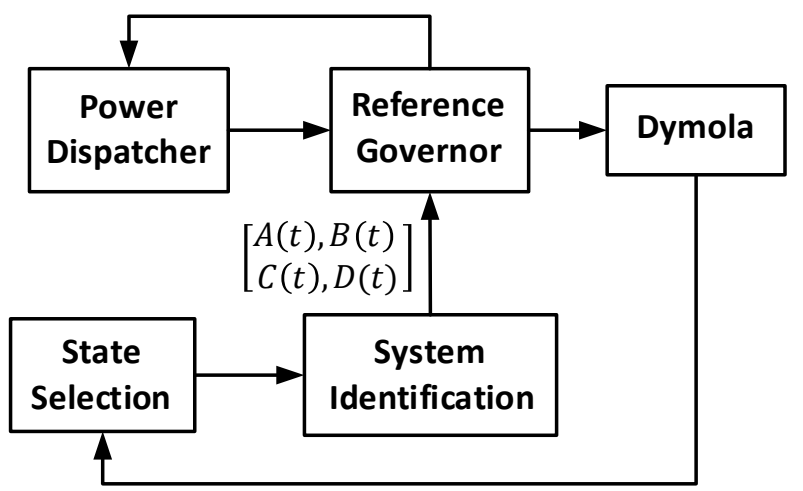

(a)

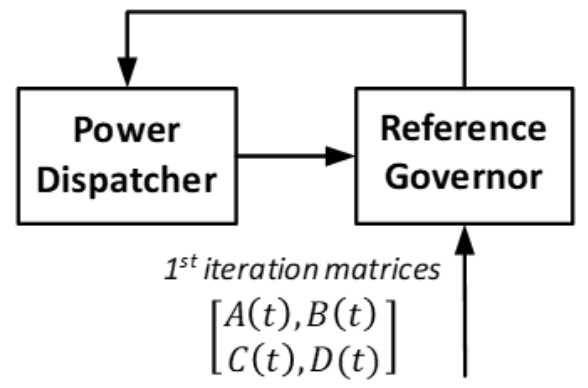

(b)

Figure 2-5. Configuration of the LTV model-based optimization scheme during the first iteration (on the left) and the following ones (on the right). 
Unlike the constant matrices scheme, the configuration of the optimization scheme will change from the first iteration to the following ones. In particular, after the first iteration, the configuration of the LTV model-based optimization scheme will become identical to the one of the LTI modelbased optimization scheme (Figure 2-5). Since the load demand profile to be met is the same, the optimized set-point trajectory after the second iteration will not be very different from the one obtained after the first iteration, i.e., a smoothed trajectory is expected. For this reason, the set of matrices identified during the first iteration can be used as a good approximation of the system dynamics during the second iteration. Accordingly, the State Selection and the System Identification algorithms will be run only during the first iteration. The outcomes of these algorithms will be re-cycled during the second iteration. In this way, the adjusted power dispatch needs to be simulated by adopting the Dymola model only during the first iteration.

Finally, a hybrid configuration between the LTI model-based and the LTV model-based ones can be foreseen. It consists of a parametrized approach (Figure 2-6). In particular, Dymola simulations will be run to get the input data, and then a set of "parametrical" matrices will be derived.

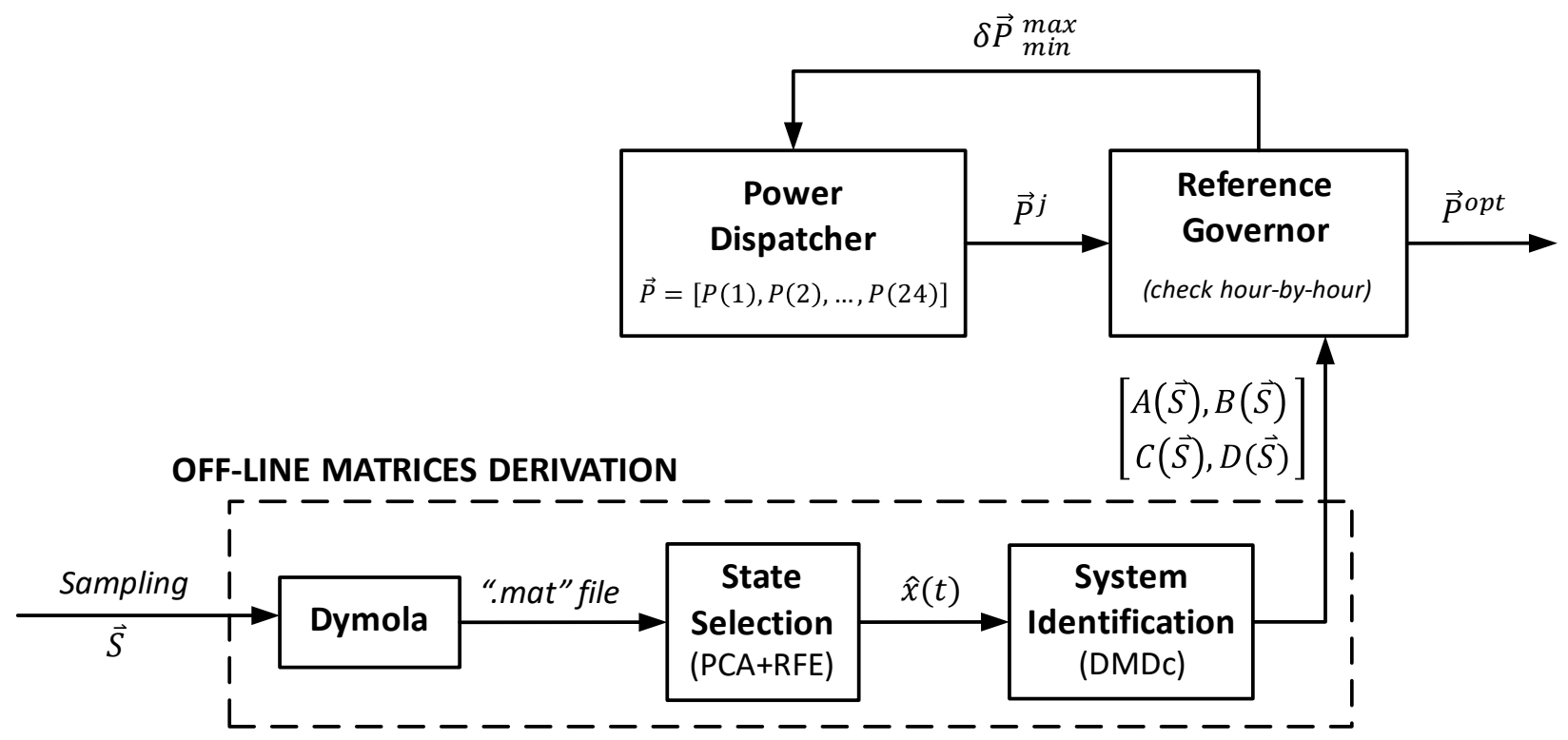

Figure 2-6. LTI model-based optimization scheme (state-space representation matrices are derived offline and parametrized).

\section{DEFINITION OF A STATE VARIABLES SELECTION ALGORITHM}

\subsection{Definition of the state of the system}

The state variables of a dynamic system are represented by the minimum set of variables that fully describe the system and its response to any given set of inputs. The dynamic behavior of a state-determined system is completely characterized by the response of the set of $n$ variables $x_{i}(t)$, where the number $n$ is defined to be the order of the system (Figure 3-1). This means that if the 
values assumed by these variables $x_{i}(t), i=1, \ldots, n$ at an initial time $t=t_{0}$ are known, together with the system inputs for time $t \geq t_{0}$, the future system states and outputs can be predicted for all time $t \geq t_{0}$. Different sets of variables can be selected to yield a complete system description. State variable descriptions of systems may be formulated in terms of physical and measurable variables, or in terms of variables that cannot be directly measured. It is possible to mathematically transform one set of state variables to another; the important point is that any set of state variables must provide a complete description of the system [6].

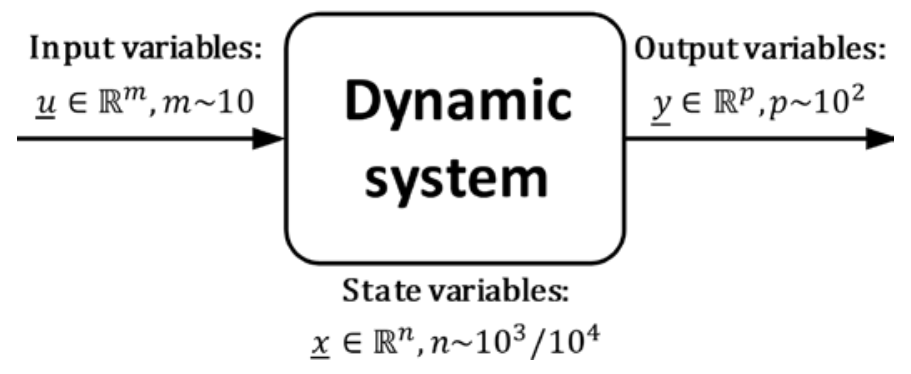

Figure 3-1. Dynamic system configuration. Input, Output and State variable are represented.

In the standard form, the mathematical description of the system is expressed as a set of $n$ coupled first-order Ordinary Differential Equations (ODEs), known as the state equations, in which the time derivative of each state variable is expressed in terms of the state variables $\vec{x}(t)=$ $\left[x_{1}(t), \ldots, x_{n}(t)\right]^{T}$ and the system inputs $\vec{u}(t)=\left[u_{1}(t), \ldots, u_{m}(t)\right]^{T}$, as shown in Eq. (1).

$$
\begin{aligned}
& \dot{x}_{1}=f_{1}(\vec{x}(t), \vec{u}(t), t) \\
& \dot{x}_{2}=f_{2}(\vec{x}(t), \vec{u}(t), t) \\
& \vdots \\
& \dot{x}_{n}=f_{n}(\vec{x}(t), \vec{u}(t), t)
\end{aligned}
$$

In case of linear, time-invariant systems (LTI), the dynamics is described by linear differential equations with constant coefficients. For an LTI system of order $n$ and with $m$ inputs, Eq.(1) become a set of $n$ coupled first-order linear differential equations with constant coefficients $\left(a_{i j}\right.$ and $b_{i j}$ ):

$$
\begin{aligned}
& \dot{x}_{1}=a_{11} x_{1}+a_{12} x_{2}+\cdots+a_{1 n} x_{n}+b_{11} u_{1} \cdots+b_{1 m} u_{m} \\
& \dot{x}_{2}=a_{21} x_{1}+a_{22} x_{2}+\cdots+a_{2 n} x_{n}+b_{21} u_{1} \cdots+b_{2 m} u_{m} \\
& \vdots \\
& \dot{x}_{n}=a_{n 1} x_{1}+a_{n 2} x_{2}+\cdots+a_{n n} x_{n}+b_{n 1} u_{1} \cdots+b_{n m} u_{m}
\end{aligned}
$$

A system output $\left(y_{i}(t), i=1, \ldots, p\right)$ is defined to be any system variable of interest. An important property of the linear state equation description is that all system variables may be represented by a linear combination of the state variables and the system inputs. 


$$
\begin{aligned}
& y_{1}=c_{11} x_{1}+c_{12} x_{2}+\cdots+c_{1 n} x_{n}+d_{11} u_{1} \cdots+d_{1 m} u_{m} \\
& y_{2}=c_{21} x_{1}+c_{22} x_{2}+\cdots+c_{2 n} x_{n}+d_{21} u_{1} \cdots+d_{2 m} u_{m} \\
& \vdots \\
& y_{p}=c_{p 1} x_{1}+c_{p 2} x_{2}+\cdots+c_{p n} x_{n}+d_{p 1} u_{1} \cdots+d_{p m} u_{m}
\end{aligned}
$$

Eqs.(2)(3) may be written compactly in a matrix form:

$$
\begin{aligned}
& \overrightarrow{\dot{x}}(t)=A \vec{x}(t)+B \vec{u}(t) \\
& \vec{y}(t)=C \vec{x}(t)+D \vec{u}(t)
\end{aligned}
$$

where $A \in \mathbb{R}^{n \times n}, B \in \mathbb{R}^{n \times m}, C \in \mathbb{R}^{p \times n}, D \in \mathbb{R}^{p \times m}$ are matrices of constant coefficients $a_{i j}$, $b_{i j}, c_{i j}$, and $d_{i j}$, respectively. Usually, LTI systems are used as an effective approximation of the governing dynamics of the studied system. On the other hand, if the matrices are not constant, a linear time-variant (LTV) approximation of the system dynamics can be obtained (Eq.(5)).

$$
\begin{aligned}
& \overrightarrow{\dot{x}}(t)=A(t) \vec{x}(t)+B(t) \vec{u}(t) \\
& \vec{y}(t)=C(t) \vec{x}(t)+D(t) \vec{u}(t)
\end{aligned}
$$

With respect to the dispatch optimization problem, the studied system is constituted by the closedloop given by the "Dymola model" and the "PID controllers", as shown in Figure 1-3. For such a system, the inputs are the set-points issued by the power dispatcher and then adjusted by the RG algorithm $(\vec{v}(t))$. In addition, the RG is a discrete time control (or "digital control") scheme. It means that the time-continuous variables entering and exiting the RG block are sampled at a certain frequency, and that the analysis is carried out in the discrete-time domain, i.e., a discrete-time model will be used to represent the controlled system dynamics. Eq.(6) shows the state-space model that is used in the RG implementation.

$$
\begin{aligned}
& \vec{x}(k+1)=A^{d} \vec{x}(k)+B^{d} \vec{v}(k) \\
& \vec{y}(k)=C^{d} \vec{x}(k)+D^{d} \vec{v}(k)
\end{aligned}
$$

\subsection{Problem description and developed two-step process}

Given the complexity of the studied system, the minimum set of variables that constitutes the state of the system cannot be analytically/numerically identified from the set of ODEs. In addition, the set of state variables might need to be updated according to the instantaneous operating conditions. If the dynamics of the studied system is linear, the variables that constitute the state of the system are always the same. This might not be true if the system is characterized by highly non-linear dynamics. In that case, the set of state variables might change. During a transient, as the system explores different operating conditions, new variables can be involved in defining the state of the system. 
For these reasons, a data driven system was then foreseen. The methodology for the deployment of an automated approach for the state variable selection problem has been defined, designed and delivered in the RAVEN framework [7][8][9]. The method has been designed based on the following methodological/functional requirements:

- Agnosticism: the methodology has to be accurate and deployable for any system model of interest

- Automation: the process must be used in an automated fashion, with minimum intervention from the user

- Performance-driven: the method must not be too computational expensive since it could be used in an online setup.

Based on the above requirements, a combination of methods has been assembled in an automated workflow in the RAVEN software. As shown in Figure 3-2, the proposed workflow is deployed in a multi-step procedure, (1) modeling of the system of interest, using Dymola, (2) feature space dimensionality reduction and projection in the reduced space, and (3) feature selection step in the reduced space. In the following sections, a brief description of the different steps is reported.

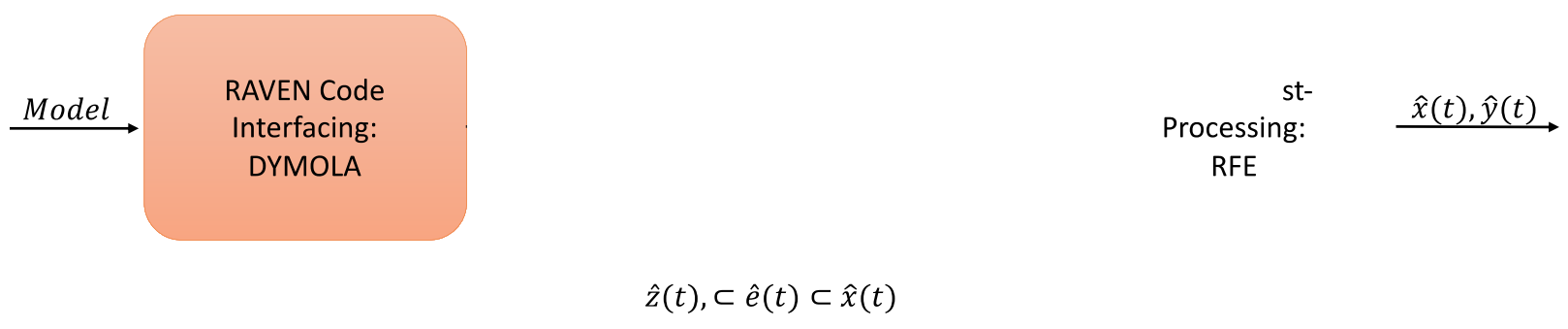

Figure 3-2. State selection process workflow in RAVEN.

\subsection{First step: System Modeling}

As anticipated, the system modeling for this project is performed using models developed under the Dymola simulation environment. When a Dymola simulation is run, a standard MATLAB binary output file (.mat) is generated. It contains the values of all the parameters and the variables involved in the equations that constitute the studied dynamic model. The trajectories of all the variables are stored in the generated .mat file.

\subsection{Second step: Principal Component Analysis}

Principal component analysis (PCA) is a technique for reducing the dimensionality of large, high-dimensional datasets (with $n$ observations on $p$ numerical variables), increasing interpretability but at the same time minimizing information loss. The approach achieves it creating new uncorrelated variables/dimensions (principal components) that maximize the 
variance within the dataset. The method basically consists in solving an eigenvalue/eigenvector problem.

Formally, the data values define a data matrix $X \in \mathbb{R}^{n \times p}$, whose $j^{\text {th }}$ column is the vector $\vec{x}_{j}$ of observations on the $j^{\text {th }}$ variable. As mentioned herein, the PCA method is aimed to seek a linear combination of the columns of matrix $X$ with maximum variance. Such linear combinations are given by

$$
\sum_{j=1}^{p} a_{j} \vec{x}_{j}=X \vec{a}
$$

where $\vec{a}$ is a vector of constants $a_{1}, a_{2}, \ldots, a_{p}$. The variance of any linear combination is given by

$$
\operatorname{var}(X \vec{a})=\vec{a}^{T} S \vec{a}
$$

where $S$ is the sample covariance matrix associated with the dataset. Hence, identifying the linear combination with maximum variance is equivalent to obtaining a p-dimensional vector $\vec{a}$ which maximizes the quadratic form $\vec{a}^{T} S \vec{a}$. To produce a defined solution to the problem, a constraint needs to be imposed; commonly, the restriction involves the requirement to work with unit-norm vectors $\left(\vec{a}^{T} \vec{a}=1\right)$. Consequentially, the problem is equivalent to the optimization of $\vec{a}^{T} S \vec{a}-\lambda\left(\vec{a}^{T} \vec{a}-1\right)$, where $\lambda$ is a Lagrange multiplier. Differentiating with respect to the vector $\vec{a}$ and equating to the null vector, produces the equation

$$
S \vec{a}=\lambda \vec{a}
$$

Thus, $\vec{a}$ must be a (unit-norm) eigenvector, and $\lambda$ the corresponding eigenvalue, of the covariance matrix $S$. Any real symmetric matrix $\in \mathbb{R}^{p \times p}$, such as a covariance matrix $S$, has exactly $p$ real eigenvalues $\lambda_{k}(k=1, \ldots, p)$, and their corresponding eigenvectors can be defined to form an orthonormal set of vectors

$$
\left\{\begin{array}{cc}
\vec{a}_{k}^{T} \vec{a}_{k^{\prime}}=1 & \text { if } k=k^{\prime} \\
0 & \text { if } k \neq k^{\prime}
\end{array}\right.
$$

The main idea using this approach in this project is to reduce the dimensionality of the feature space still capturing the main drivers in the feature dataset.

The PCA method is applied on the feature space only (no output variables) for the construction of a new reduced dataset. The number of PCA components to be selected is currently manually evaluated according to the accuracy. At the same time, the goal is to make this process automated. 


\subsection{Third step: Recursive Feature Elimination}

One of the problems faced in the machine learning realm is to find ways to reduce the dimensionality of the feature space to overcome the risk of "overfitting" and improve the general performance of the models. Performing feature selection in large dimensional input spaces generally involves greedy algorithms. Among various possible methods feature-ranking techniques are particularly attractive, since they are aimed to identify a fixed number of top ranked features to be selected for construction of representative models and/or identification of important dimensions/inputs for the figure of merits of interest. Among the different available approaches, the Recursive Feature Elimination (RFE) and its variant Cross-Validated Recursive Feature Elimination (CVRFE) [10] has been deployed in the RAVEN framework for the development of this project. The method consists of the following iterative procedure:

1. Construct an evaluation model (e.g., linear model), trained on a complete feature space dataset;

2. Compute a ranking criterion for all features by using:

2.1. Sensitivity ranking method (ranking of the features based on their sensitivity coefficients)

2.2. Correlation ranking approach (ranking of the features based on their correlation coefficients)

3. Remove the feature with smallest ranking criterion.

This iterative procedure is an instance of backward feature elimination. For computational reasons, it may be more efficient to remove several features at a time, at the expense of possible classification performance degradation. In such a case, the method produces a feature subset ranking, as opposed to a feature ranking. Feature subsets are nested $F_{1} \subset F_{2} \subset F_{3} \ldots \subset F$. If features are removed one at a time, there is also a corresponding feature ranking. However, the features that are top ranked (eliminated last) are not necessarily the ones that are individually most relevant. Only taken together the features of a subset $\vec{F}_{m}$ are optimal in some sense.

\section{DEFINITION OF THE SYSTEM IDENTIFICATION ALGORITHM}

Once the state variables are extracted from the Dymola-generated simulation outcomes, the state-space model of the system to be controlled can be derived. Given the iterative nature of the proposed optimization process, the efficiency represents the main requirement in the choice of the most suitable algorithm. The Dynamics Mode Decomposition with Control (DMDc) [11] was adopted. Dynamic mode decomposition (DMD) is a dimensionality reduction algorithm developed by P. Schmid in 2008 [12]. Given a time series of data, DMD computes a set of modes each of which is associated with a fixed oscillation frequency and decay/growth rate. DMDc is a modification of the traditional DMD procedure. It allows distinguishing between the underlying dynamics and the influence of the system inputs, resulting in accurate input-output models, i.e., 
DMDc algorithm allows obtaining the $A^{d}$ and $B^{d}$ matrices of a state-space representation of the monitored system dynamics. Since the derived matrices represent the best-fit solutions for the data contained in the training set, the relationship in Eq.(6) does not hold exactly:

$$
\vec{x}(k+1) \approx A^{d} \vec{x}(k)+B^{d} \vec{v}(k)
$$

State-space model matrices can be constructed by collecting temporal snapshots of the system states and inputs over time (Eq.(12)).

$$
\begin{aligned}
& \mathrm{X}^{\prime}=\left[\begin{array}{cccc}
\mid & \mid & & \mid \\
x(2) & x(3) & \cdots & x(l) \\
\mid & \mid & & \mid
\end{array}\right] \\
& \mathrm{X}=\left[\begin{array}{cccc}
\mid & \mid & \mid \\
x(1) & x(2) & \cdots & x(l-1) \\
\mid & \mid & & \mid
\end{array}\right] \\
& \mathrm{U}=\left[\begin{array}{cccc}
\mid & \mid & & \mid \\
v(1) & v(2) & \cdots & v(l-1) \\
\mid & \mid & & \mid
\end{array}\right]
\end{aligned}
$$

where each column represents the system state $(\vec{x}(k))$ or input arrays $(\vec{v}(k))$ evaluated or measured at a certain time step. Eq.(11) can be rewritten in a matrix form to include the new data matrices:

$$
\mathrm{X}^{\prime} \approx A^{d} \mathrm{X}+B^{d} \mathrm{U}
$$

Since there are two unknown matrices $A^{d}$ and $B^{d}$ in the equation, Eq.(13) can be rewritten as:

$$
\mathrm{X}^{\prime} \approx G \Omega
$$

where $G=\left[A^{d}, B^{d}\right]$ and $\Omega=[X, U]^{T}$. Thus, the operator $G \in \mathbb{R}^{n \times(n+m)}$ can be calculated as:

$$
G=\mathrm{X}^{\prime} \Omega^{\dagger}
$$

To solve Eq.(15), the Singular Value Decomposition (SVD) is applied to the augmented data matrix $\Omega$, which leads to:

$$
\Omega=U \Sigma \mathrm{V}^{*} \approx \widetilde{\mathrm{U}} \widetilde{\Sigma} \widetilde{\mathrm{V}}^{*}
$$

where $\widetilde{\mathrm{U}} \in \mathbb{R}^{(n+m) \times q}, \tilde{\Sigma} \in \mathbb{R}^{q \times q}$, and $\widetilde{\mathrm{V}} \in \mathbb{R}^{(l-1) \times q}$ are the truncated SVD components.

The truncation value $q$ is the number of non-zero elements in $\Sigma$ by default, but it can be manually defined to a smaller value if desired. Eq.(17) provides an approximation of $G$ :

$$
G \approx \bar{G}=\mathrm{X}^{\prime} \widetilde{\mathrm{V}} \widetilde{\Sigma}^{-1} \widetilde{\mathrm{U}}^{*}
$$


By breaking the linear operator $\widetilde{\mathrm{U}}$ into two separate components according to the dimensions of $\vec{x}(k)$ and $\vec{v}(k)$, the approximations of the matrices $A^{d}$ and $B^{d}$ can be found (Eq.(18)).

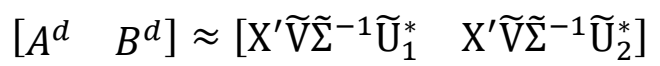

where $\widetilde{\mathrm{U}}_{1} \in \mathbb{R}^{n \times q}$ and $\widetilde{\mathrm{U}}_{2} \in \mathbb{R}^{m \times q}$.

To complete the definition of the state-space representation model (Eq.(6)), matrices $C^{d}$ and $D^{d}$ need to be derived as well. These matrices express the evolution of the output variables $(\vec{y}(k))$ as a linear combination of the state variables and the input variables. In particular, $C^{d}$ matrix represents the influence of $\vec{x}(k)$, and $D^{d}$ represents the influence of $\vec{v}(k)$. Based on the nature of the systems to be studied, the input vector has no direct impact to the system output, i.e., $D^{d}$ matrix is zero (Eq.(19)).

$$
D^{d}=0
$$

To this end, the output equation in Eq.(6) reduces to:

$$
\vec{y}(k)=C^{d} \vec{x}(k)
$$

To find the best-fit solution of the $C^{d}$ matrix using the system state vectors $\vec{x}(k)$ and system output vectors $\vec{y}(k)$ in the training data set, the matrix of temporal snapshot of system output vector $\vec{y}(k)$ is constructed (Eq.(21)).

$$
Y=\left[\begin{array}{cccc}
\mid & \mid & & \mid \\
y(1) & y(2) & \cdots & y(l-1) \\
\mid & \mid & & \mid
\end{array}\right]
$$

Eq. (20) can be rewritten in a matrix form by inserting the state variable $(\mathrm{X})$ and the output variable (Y) snapshots:

$$
\mathrm{Y}=C^{d} \mathrm{X}
$$

$C^{d}$ matrix can then be approximated as:

$$
C^{d} \approx \mathrm{YX}^{-1}
$$

In particular, Moore-Penrose pseudo inverse algorithm [13] is used to invert $X$ in Eq.(23). In addition, the accuracy of $C^{d}$ will increase with the diversity of $X$ in the training data. 


\section{DEFINITION OF THE REFERENCE GOVERNOR ALGORITHM}

Each one of the IES unit components can be modeled as a Single Input Multiple Output system. The corresponding models receives as input the adjusted set-point trajectory $(v)$, and returns as outputs the monitored process variables $(\vec{y})$, as shown in Figure 1-3. The goal of the RG algorithm placed upstream to the Dymola models is generating from the reference scalar signal $(r)$ a feasible set-point signal $(v)$ that meets all the constraints on the monitored variables.

In this section, the equations that constitute the Single Input Multiple Output version of the RG algorithm are reported. Let us consider a system characterized by $p$ output variables $(\vec{y}=$ $\left[y_{1}, y_{2}, \ldots, y_{p}\right]^{T}$ ), and let us impose a lower and an upper limit to each one of these variables. These bounds can be represented by a set of linear inequalities, i.e., $y_{1} \geq y_{1}{ }^{\min }$ and $y_{1} \leq y_{1}{ }^{\max }$. For simplicity, let us assume that these constraints are constant (Eq.(24)).

$$
\left[\begin{array}{cccc}
+1 & 0 & \cdots & 0 \\
-1 & 0 & \cdots & 0 \\
0 & +1 & \cdots & 0 \\
0 & -1 & \cdots & 0 \\
\vdots & \vdots & \ddots & \vdots \\
\vdots & \vdots & \ddots & \vdots \\
0 & 0 & \cdots & +1 \\
0 & 0 & \cdots & -1
\end{array}\right] \cdot\left[\begin{array}{c}
y_{1} \\
y_{2} \\
\vdots \\
y_{p}
\end{array}\right] \leq\left[\begin{array}{c}
+y_{1} \max \\
-y_{1} \min \\
+y_{2} \max \\
-y_{2} \min \\
\vdots \\
+y_{p} \max \\
-y_{p} \min
\end{array}\right] \Rightarrow S \cdot \vec{y} \leq \vec{s}
$$

The RG algorithm is designed to ensure that imposed linear inequalities are always met during both steady state and transient operation. RG, like the Model-based Predictive Control, is based on iterative, finite-horizon optimization scheme. At time $t=k$, the current plant state is sampled and the optimal set-point trajectory is calculated for a relatively short time horizon $t \in$ $[k, k+g]$. Specifically, an online calculation is used to explore state trajectories that evolve from the current state and find a cost-minimizing control strategy until time $t=k+g$. Only the first step of the control strategy is implemented, then the plant state is sampled again and the calculations are repeated starting from the new current state, yielding a new trajectory and new predicted state path. Since the prediction horizon is continuously shifted forward, RG is also called receding horizon control scheme.

When constant input signals are supplied $(v(k)=v)$, the state variables do not change $(\vec{x}(k)=$ $\vec{x})$. Let us define the matrix $T$ as the ratio between the state of the system and the corresponding inputs (Eq.(26)).

$$
\begin{aligned}
& \vec{x}=A^{d} \cdot \vec{x}+B^{d} \cdot v \\
& T=\frac{\vec{x}}{v}=\left(I-A^{d}\right)^{-1} \cdot B^{d}
\end{aligned}
$$


By adopting Eqs.(6)(26), the linear inequalities in Eq.(24) can be rewritten at steady state as follows:

$$
S \cdot \vec{y}=S C^{d} T v+S D^{d} v \leq \vec{s}
$$

By defining a suitable matrix Lim (Eq.(28)), the linear inequalities to be satisfied when a constant input signal is supplied can be expressed as shown in Eq.(29).

$$
\begin{aligned}
& \operatorname{Lim}=S \cdot\left(C^{d} T+D^{d}\right) \\
& 0 \cdot \vec{x}(k)+\operatorname{Lim} \cdot v(k) \leq \vec{S}
\end{aligned}
$$

Similarly, for time-dependent input signals, the RG algorithm is meant to verify that output variables will not assume forbidden values over a certain time horizon as a result of an imposed set-point. To this aim, let us first verify that constraints are not violated at the current time step $(k)$ :

$$
S \cdot \vec{y}(k)=S \cdot C^{d} \cdot \vec{x}(k)+S \cdot D^{d} \cdot v(k) \leq \vec{s}
$$

By defining matrices $K_{x}$ and $K_{r}$ (Eq.(31)), the linear inequality to be satisfied can be re-written as shown in Eq.(32).

$$
\begin{aligned}
& K_{x}=S C^{d}, K_{r}=S D^{d} \\
& K_{x} \cdot \vec{x}(k)+K_{r} \cdot v(k) \leq \vec{s}
\end{aligned}
$$

Similarly, the set-point feasibility needs to be assessed for the following time steps, i.e., the states evaluated at time $t=k+1, k+2, \ldots, k+g$ need to satisfy Eq.(32), where $g$ is an positive integer representing the length of the considered time horizon.

$$
K_{x} \cdot \vec{x}(k+m)+K_{r} \cdot v(k) \leq \vec{s}
$$

where $m=1,2, \ldots, g$. To evaluate the evolution of the state variables from the current conditions $(\vec{x}(k))$, state space representation matrices are adopted (Eq.(34)).

$$
\begin{aligned}
& S C^{d} \cdot \vec{x}(k+m)+S D^{d} \cdot v(k) \leq \vec{s} \\
& S C^{d}\left(\mathrm{~A} \cdot \vec{x}(k+m-1)+B^{d} \cdot v(k)\right)+S D^{d} \cdot v(k) \leq \vec{s} \\
& S C^{d} \mathrm{~A} \cdot \vec{x}(k+m-1)+S C^{d} B^{d} \cdot v(k)+S D^{d} \cdot v(k) \leq \vec{s} \\
& S C^{d} \mathrm{~A} \cdot \vec{x}(k+m-1)+S\left(C^{d}\left(I-A^{d}\right)\left(I-A^{d}\right)^{-1} \cdot B^{d}+D^{d}\right) \cdot v(k) \leq \vec{s} \\
& S C^{d} \mathrm{~A} \cdot \vec{x}(k+m-1)+S\left(C^{d}\left(I-A^{d}\right) T+D^{d}\right) \cdot v(k) \leq \vec{s} \\
& S C^{d} \mathrm{~A} \cdot \vec{x}(k+m-1)+S\left(C^{d} T+D^{d}-C^{d} A T\right) \cdot v(k) \leq \vec{s} \\
& K_{x} \mathrm{~A} \cdot \vec{x}(k+m-1)+\left(\operatorname{Lim}-K_{x} A T\right) \cdot v(k) \leq \vec{s}
\end{aligned}
$$


In particular, Eq.(35) shows the linear inequalities that $\vec{x}(k+1)$ and $v(k)$ should satisfy when $m=1$ :

$$
K_{x} \mathrm{~A} \cdot \vec{x}(k)+\left(\operatorname{Lim}-K_{x} A T\right) \cdot v(k) \leq \vec{s}
$$

Similarly, Eq.(36) shows the linear inequalities for $\vec{x}(k+g)$ :

$$
K_{x} A^{g} \cdot \vec{x}(k)+\left(\operatorname{Lim}-K_{x} A^{g} T\right) \cdot v(k) \leq \vec{s}
$$

In this way, the linear inequalities that current system state $\vec{x}(k)$ and current actuation $v(k)$ need to satisfy over the imposed time horizon can be obtained. In Eq.(37), the constraints expressed in Eq.(24) evaluated at a certain time step from $t=k$ to $t=k+g$ are reported. In Eq.(38), the corresponding matrix equation is reported.

$$
\begin{aligned}
& {\left[\begin{array}{cc}
0 \cdot K_{x} & \operatorname{Lim} \\
K_{x} & K_{r} \\
K_{x} A & \operatorname{Lim}-K_{x} A T \\
K_{x} A A & \operatorname{Lim}-K_{x} A A T \\
\ldots & \ldots \\
K_{x} A^{g} & \operatorname{Lim}-K_{x} A^{g} T
\end{array}\right] \cdot\left[\begin{array}{l}
\vec{x}(k) \\
v(k)
\end{array}\right] \leq\left[\begin{array}{c}
\vec{s} \\
\vec{s} \\
\vec{s} \\
\vec{s} \\
\cdots \\
\vec{s}
\end{array}\right]} \\
& H_{x} \cdot \vec{x}(k)+H_{v} \cdot v(k) \leq \vec{h}
\end{aligned}
$$

The set of linear inequalities reported in Eqs.(37)(38) is called Maximal Output Admissible Set (MOAS). A $v(k)$ satisfying the MOAS ensures that the system output will not exceed the upper or lower bounds both during transient and at final steady-state. This ensure that the modified reference command is as close as possible to the original set-points, the scalar value $\beta(k)$ is maximize within the range [0,1] by respecting the linear inequalities $H_{x} \cdot \vec{x}(k)+H_{v} \cdot \vec{v}(k) \leq \vec{h}$ in MOAS:

$$
v(k)=v(k-1)+\beta(k)[r(k)-v(k-1)]
$$

where $\beta(k) \in[0,1], r(k)$ is the input set-point issued at this time step, and $v(k-1)$ is the adjusted set-point at the previous time step. In the case when $r(k)$ and $v(k)$ are scalar values, the admissible range of $v(k)$ can be found by calculating the union of each row in the linear inequalities $H_{x} \cdot x(k)+H_{v} \cdot v(k) \leq \vec{h}$.

As above-mentioned, the presented version of the RG optimizes the value of a scalar variable $(\beta(k))$ to adjust the input set-point $(r(k))$. At the same time, other versions of the RG are available. In particular, when Multiple Input Multiple Output (MIMO) systems are considered, multiple setpoint signals can be simultaneously optimized. In this case, more complicate systems and 
interactions can be addressed, but the solution of the optimization problem would require quadratic programming techniques. For further information, the reader may refer to [5][14][15][16].

\section{VALIDATION OF THE DEVELOPED OPTIMIZATION SCHEME}

\subsection{Test case description}

To assess the developed optimization scheme, a test-case was developed. Given that there is no reference configuration for the IES system, a two-unit system constituted by the Balance of Plant of the Nuclear unit (BOP), and the Secondary Energy System (SES), was assembled. In Table 6-1, the monitored output variables for each one unit, and the corresponding upper and lower bounds are reported.

Table 6-1. Selected components for the developed test-case. The main constraints and the corresponding operational bounds are reported for each one of them.

\begin{tabular}{cll}
\hline IES Unit component & Constrained process variables & \multicolumn{1}{c}{ Variable Constraints } \\
\hline \multirow{2}{*}{ Balance of Plant } & - Electrical power $\left(P_{B O P}\right)$ & $\bullet[50 \%, 100 \%] \cdot 1.45 \times 10^{9} \mathrm{~W}$ \\
& $\bullet$ Steam turbine inlet pressure $\left(p_{B O P}\right)$ & $\bullet[65.8,67.8 \mathrm{bar}]$ \\
\multirow{2}{*}{ Secondary Energy System } & $\bullet$ Electrical power $\left(P_{S E S}\right)$ & $\bullet[30 \%, 100 \%] \cdot 45 \times 10^{6} \mathrm{~W}$ \\
& $\bullet$ Gas turbine firing temperature $\left(T_{S E S}\right)$ & $\bullet\left[816^{\circ} \mathrm{C}, 1600{ }^{\circ} \mathrm{C}\right]$ \\
\hline
\end{tabular}

The upper and lower bounds of the constrained process variables are selected based on the practical experience and material limits.

As for the BOP, the minimum requirements for the flexible operation capabilities of modern reactors are defined by the utility requirements, which are based on the requirements of the grid operators. In particular, nuclear units must be capable of a minimum daily load cycling operation between $50 \%$ and $100 \%$ of their nominal power output [16]. At the same time, there are other process variables need to be constrained, i.e., the steam pressure at the turbine inlet, and the steam quality at the turbine outlet. In [17], the case of a BWR steam turbine is described. In particular, the turbine inlet pressure at nominal conditions is equal to 950 psig ( $65.5 \mathrm{bar}$ ). The minimum allowed value for this variable during normal operation is set equal to 920 psig (64.12 bar). With respect to the studied $\mathrm{BOP}$, we can then impose a very limited operational margin on the turbine inlet pressure variation (2 bar). Finally, a minimum value should be imposed to the steam quality at turbine outlet to limit the erosion of intermediate and low-pressure blades (presence of water droplets in the steam flow rate) [16]. For simplicity, this constraint will not be considered in the present analysis.

As for the SES, the minimum allowed electrical power output can be set at 30\%. As for the upper bound, we assumed that $5 \mathrm{MW}$ overpower transients are tolerable. Another constrained process variable is the temperature at the turbine inlet, i.e., the highest temperature reached in the Brayton cycle (firing temperature). As reported in [16], given a nominal value equal to $1,340{ }^{\circ} \mathrm{C}$, the maximum bound is set equal to $1,600{ }^{\circ} \mathrm{C}$ (structural limits) and the minimum is set equal to $816^{\circ} \mathrm{C}$ (limit on carbon monoxide production). 


\subsection{Implementation in RAVEN simulation environment: simulation outcomes}

To demonstrate the performance of the proposed optimization scheme, the LTI model-based workflow (Figure 2-2) was implemented in RAVEN simulation environment. The matrices needed by the RG algorithm are derived off-line and supplied as input parameters. As mentioned in Section 6.1, the developed test-case is constituted by a two unit-system, i.e., a 1,450 MW BOP and a 45 MW SES. The test case mimicked an electricity market with a constant load demand (1460 MW) that the two committed units try to meet. In particular, the BOP has a constant generation cost of 100.0 \$/MWh, while the SES has a time-variant generation cost between 50.0 \$/MWh and 150.00 \$MWh, as shown in Figure 6-1a.

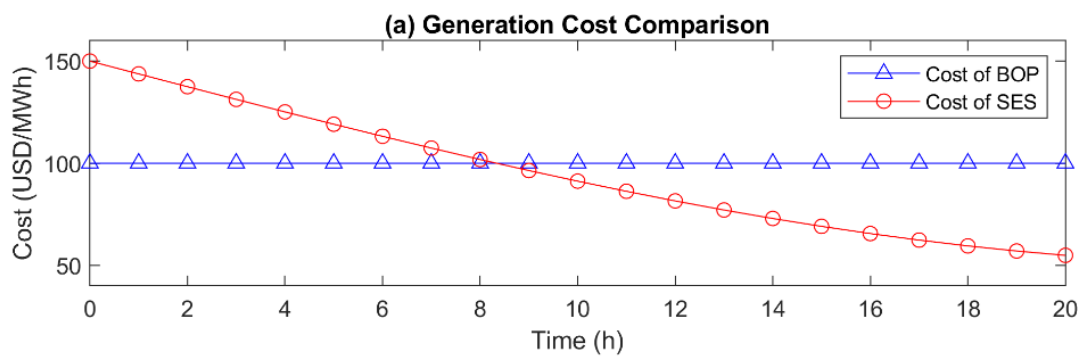

(b) BOP Power SetPoint

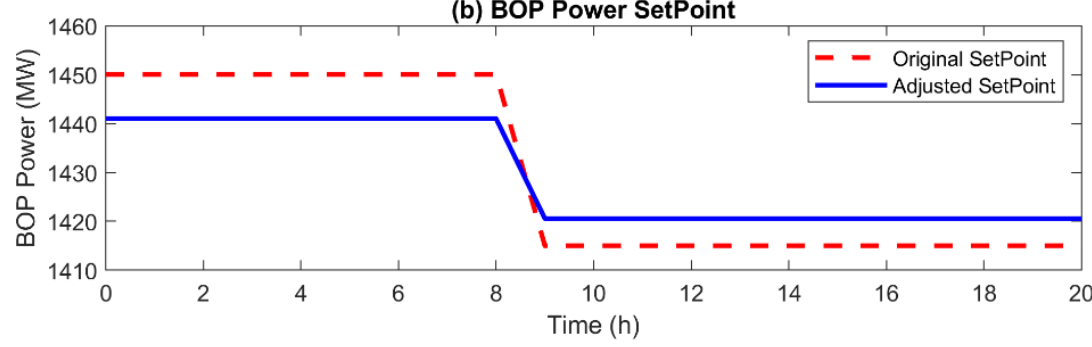

(c) SES Power SetPoint

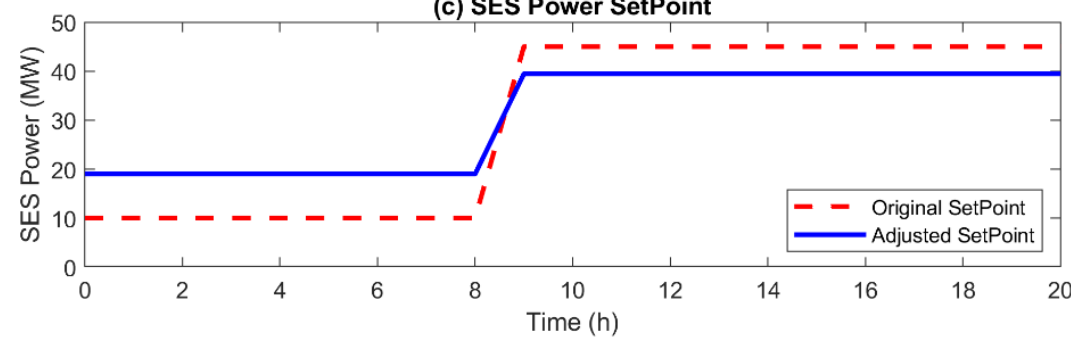

(d) SES Firing Temperature

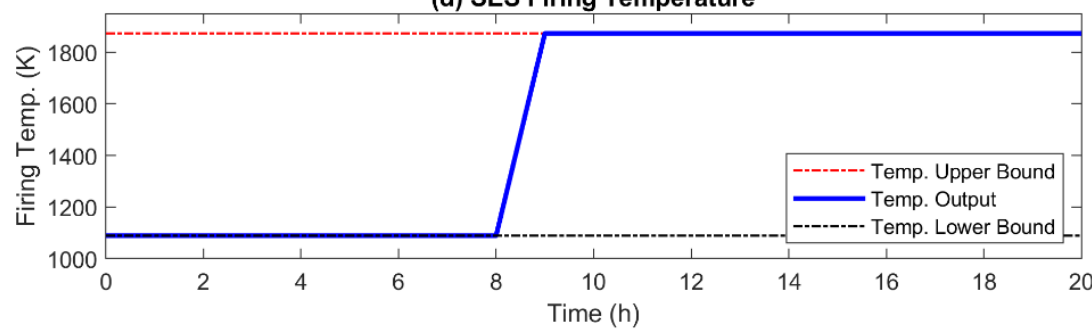

Figure 6-1. Dispatching test-case power units according to corresponding costs and thermomechanical constraints. 
The power dispatcher issues the power setpoints to each unit by minimizing the total generation cost. Initially, most of the demand is met by the BOP since its cost is lower than SES. Eventually, the SES contribution increases since its generation cost drops. The dispatcher will request the cheaper power source to operate at the corresponding full capacity, as shown by the red dashed line in Figure 6-1b and Figure 6-1c. In particular, the power dispatcher only accounts for the thermo-mechanical constraints of the SES, i.e., a single RG plugin was implemented, and it supplies the dispatcher with the SES power margins. The BOP can update its power output as long as it does not violate the electrical power bounds.

Accordingly, the RG will adjust the power set-points to ensure the SES constraints are not violated, as shown by the blue solid line in Figure 6-1c and Figure 6-1d. For instance, the SES setpoint was adjusted from 10.0 MW to 19.02 MW at the beginning of simulation, ensuring the gas turbine firing temperature is above $816^{\circ} \mathrm{C}$ to avoid the carbon monoxide production. In the second half of simulation, SES setpoint was adjusted from 45.00 MW to 39.44 MW ensuring the gas turbine firming temperature is below the structural limit of $1,600{ }^{\circ} \mathrm{C}$.

\section{DEVELOPMENT OF A CONTROL SYSTEMS MODELICA LIBRARY}

\subsection{Definition of the "Plug-and-Play" concept for control-oriented modeling}

In the context of Integrated Energy Systems, the concept of "Plug-and-Play" consists in the complete flexibility of selecting multiple power plants (e.g., gas turbine, balance of plant, thermal storage, etc.) to build a composite unit into the IES framework. At the same time, the possibility of controlling each one of the unit components with a particular control scheme (e.g., PID controllers, Model-based Predictive Controllers (MPC), Reference Governor, Kalman Filter, etc.) is ensured. In a "Plug-and-Play" simulation environment, dedicated libraries for both power plants models and control schemes as modules should be available, and the user should be allowed to selected items from both and connect them together. Modelica modeling language can ensure these capabilities [18]. At the same time, the interfaces of the developed plant models need to be modified to ensure the integration with multiple units, and the possibility of adopting different control schemes.

In Figure 7-1, the current configuration of the SES component is shown. A detailed view of the graphical user interface is shown to the right. The control scheme governing the evolution of the plant model is contained in the block labelled "Control" at the top of this picture. It is a classical PID controller that generates a signal adjusting the turbine gas flow rate. This control signal, along with the turbine power output and the gas flow rate at the turbine inlet, is represented by a dashed trajectory. Two dedicated system buses are implemented to collect these signals, i.e., the "sensorBus" and the "actuatorBus". The former collects the process variables whose evolution needs to be controlled (output variables, red dashed trajectories). The latter collects the signals that are sent to the system actuators (green dashed trajectories). 

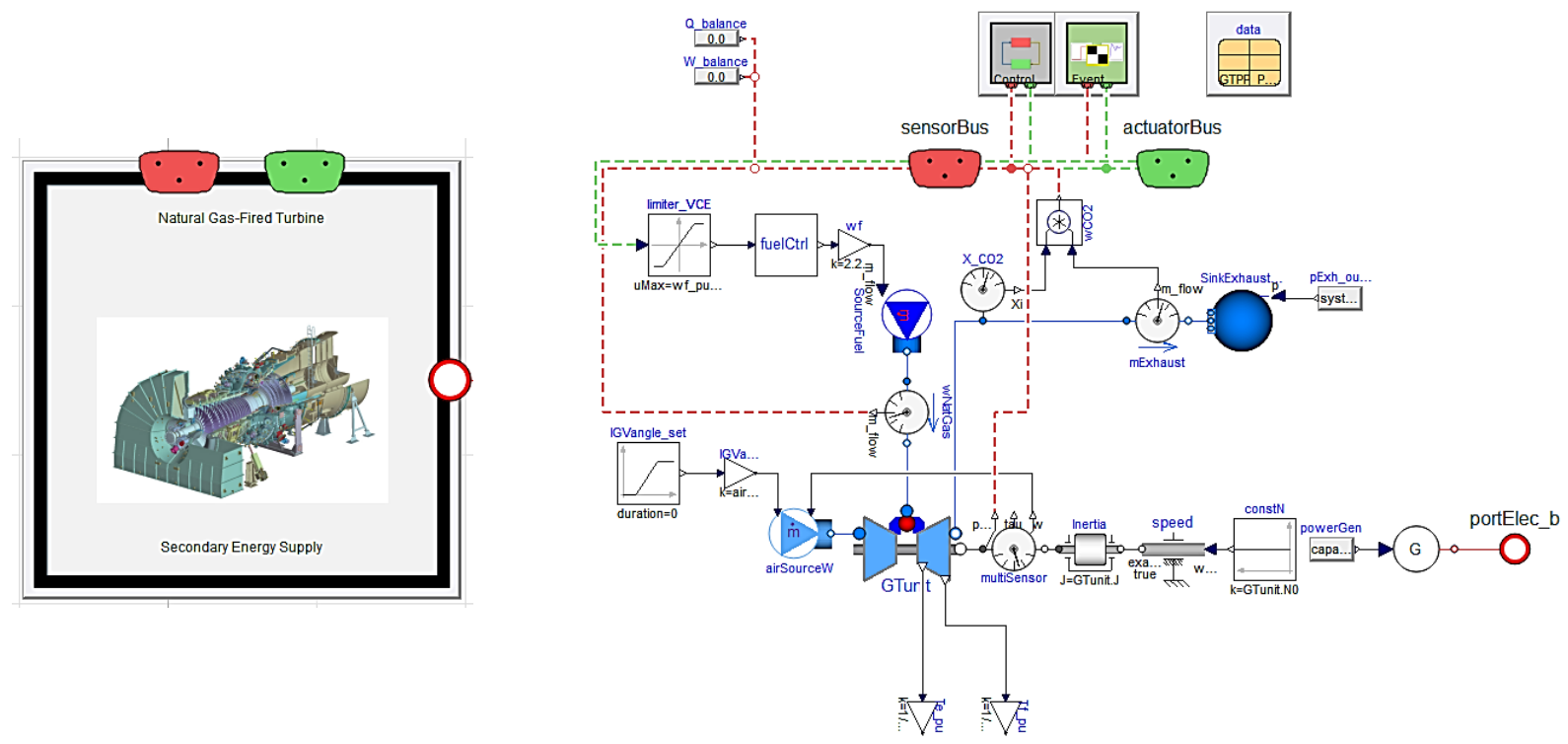

Figure 7-1. Graphical interface of the Dymola SES model (left), detailed view (right).

The encapsulation of the control scheme into a dedicated block is consistent with the "plugand-play" philosophy. In this way, the modularity of the developed model is ensured. In case one of the implemented PID controllers needs to be replaced, the user can modify the control system block, without altering the plant model. On the other hand, sensor buses are not consistent with the "plug-and-play" philosophy. If sensor buses are used, the set of variables that can be involved into the control problem is fixed. In case a new control strategy is implemented, the user needs to update the configuration of the sensorBus to account for the presence of additional items to the list of sensorBus variables.

This scenario occurs when advanced control schemes are adopted. Let us consider the implementation of the MPC [19] to control the nuclear unit. In case a core power $(P)$ drop is demanded, besides inserting the control rods $\left(\rho_{C R}\right)$, we can take advantage of the thermal reactivity feedbacks [20]. By purposefully engineering the primary circuit flow rate $\left(W_{1}\right)$ and the core inlet temperature $\left(\delta T_{i n}\right)$, faster power transients could be performed, and less abrupt temperature variations would be induced in the primary circuit.

$$
\rho_{\text {net }}=A(P-1)+B\left(P / W_{1}-1\right)+C \delta T_{\text {in }}+\rho_{C R}
$$

As shown in Figure 7-2, the three reactivity contributions $\left(\rho_{C R}, W_{1}, \delta T_{i n}\right)$ need to be calibrated and synchronized by operating the corresponding actuators, i.e., the control rods, the primary pump, which determines the primary circuit flow rate, and the feedwater pump in the BOP, which affects the core inlet temperature. The Multiple Input Multiple Output version of the MPC algorithm allows optimizing and coordinating the different actuators to achieve the desired power transient. This example demonstrates that there are control algorithms that uses several process variables to derive the optimal control actions to be performed. To implement this control 
algorithm in the current IES model, the user needs to modify both the sensorBus and the actuatorBus to collect the needed process variables.

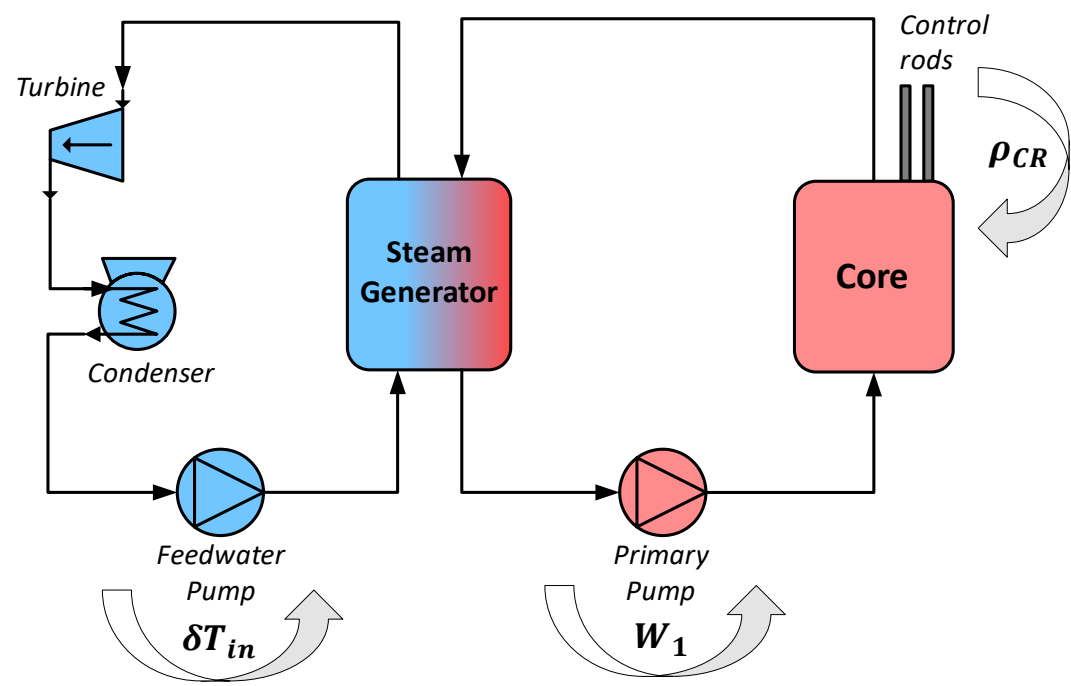

Figure 7-2. Graphical representation of a nuclear power plant operation by adopting a MIMO control scheme [20].

Large systems are traditionally provided with sensor buses that collect input and output variables signals. In this way, the value of the main process variables is made available to the supervisory control system that coordinates the operation of the different unit components. However, that is not the case for the IES. There is no supervisory control scheme in IES, nor decision-making capabilities are implemented at Dymola level. The interactions among the unit components are taken into account by HERON optimization algorithm. Each one of the implemented control schemes governs the evolution of the corresponding unit component. Regarding the present application, the adoption of system buses is counterproductive, since they do not bring any advantage in terms of plant controllability. In addition, to collect the relevant process variables into the sensorBus, duplicates of the local variables need to be defined, and this leads to an undesired proliferation of the process variables.

In the perspective of ensuring the flexibility of the Dymola modeling framework, the sensor buses should be removed. The user must be ensured the possibility of redefining the process variables to be used in the developed control strategy. It is worth reminding that the proposed modifications exclude the physical interfaces of the IES unit components. Let us consider the graphical user interface of the Balance of Plant model represented in Figure 7-3. The model of this component has both physical interfaces, which represent electrical power and water/steam flows, and non-physical interfaces, which represent control signals and measurements signals, i.e., the "sensorBus" and the "actuatorBus". To this aim, the proposed interface modification does not affect the physical interfaces. 


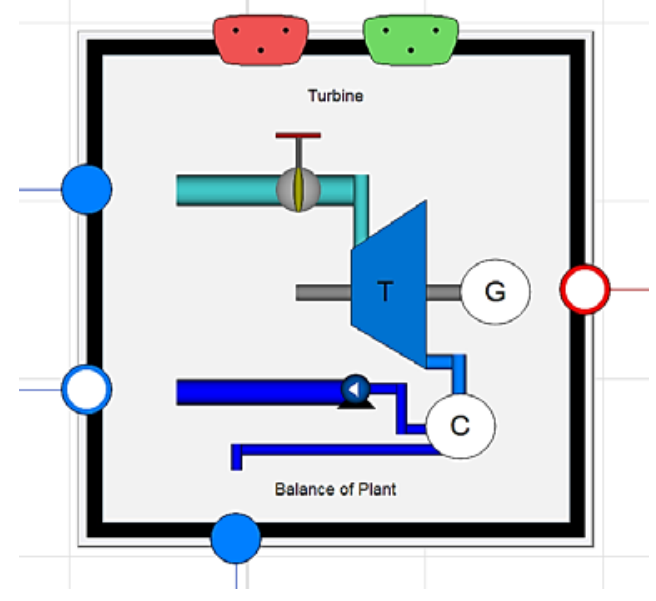

Figure 7-3. Dymola graphical interface of the BOP model component.

\subsection{List of the developed control schemes}

In Table 7-1, the developed control schemes for the new Modelica library that provides control system elements as modules are listed. The MIMO Command Governor is a high-dimensional expansion of the SISO Reference Governor, i.e., it allows adjusting an array of actuation signals. Both the SISO Reference Governor and MIMO Command Governor require the system state vector as an input, thus it must be used in combination with Kalman Filter when full-state measurement is not available. The Kalman Filter is an algorithm to estimate the system state vector by observing the input and output variables of the system, and it is essential when the system state vector is not observable. On the other hand, the DMDc algorithm needs the full-state measurement together with the actuation vector and output vector to calculate the system state space representation matrices. These available algorithms can be used in combination based on the actual variable availability.

Table 7-1. Description of the developed control schemes.

\begin{tabular}{|c|c|c|}
\hline Control Scheme & Function & Requirement \\
\hline $\begin{array}{c}\text { SISO Reference } \\
\text { Governor }\end{array}$ & $\begin{array}{l}\text { - Adjust the scalar input variable } \\
\text { - Provide the admissible range of the } \\
\text { input variable }\end{array}$ & $\begin{array}{l}\text { - } \text { System state space representation } \\
\text { matrices }(A, B, C, D) \\
\text { - } \quad \text { Current system state vector } \vec{x} \\
\text { - } \text { Constraints on outputs } y_{\min }, y_{\max }\end{array}$ \\
\hline $\begin{array}{l}\text { MIMO Command } \\
\text { Governor }\end{array}$ & $\begin{array}{l}\text { - Adjust the multi-dimension input } \\
\text { variable array } \\
\text { - Provide the of the feasible region for } \\
\text { the actuation vector }\end{array}$ & $\begin{array}{l}\text { - } \text { System state space representation } \\
\text { matrices }(A, B, C, D) \\
\text { - } \quad \text { System state vector } \vec{x} \\
\text { - } \quad \text { Constraints on outputs } y_{\min }, y_{\max }\end{array}$ \\
\hline Kalman Filter & - Estimate the system state vector & $\begin{array}{l}\text { - System state space representation } \\
\text { matrices }(A, B, C, D) \\
\text { - System actuation vector } \\
\text { - System output vector }\end{array}$ \\
\hline
\end{tabular}




Dynamic Mode
Decomposition with
Control (DMDc)

- Update the System state space representation matrices $(A, B, C, D)$
- History of system state vector

- History of system actuation vector

- History of system output vector $\vec{y}$

\subsection{Testing of the Plug-and-play configuration on SES model}

To test the proposed plant model interface and the developed control scheme library, one of the plant models in the RAVEN-based HYBRID framework was reworked accordingly. In Figure 7-4, the modified graphical user interface of the SES plant model is shown. The sensorBus and the actuatorBus were removed, and replaced with dedicated Dymola input and output interface icons, i.e., blue triangles and white triangles, to mark the control variable $(v)$, i.e., the fuel flow rate, and the process variables of interest, i.e., the electrical power output $\left(y_{1}\right)$ and the gas temperature at the turbine inlet $\left(y_{2}\right)$. As mentioned in Section 7.1, the proposed reworking does not affect the physical interfaces, only the input and output variable interface. For this reason, the physical interface connecting the SES generator with the system electric switch (the red flange labelled with "portElec_b”) was not modified.

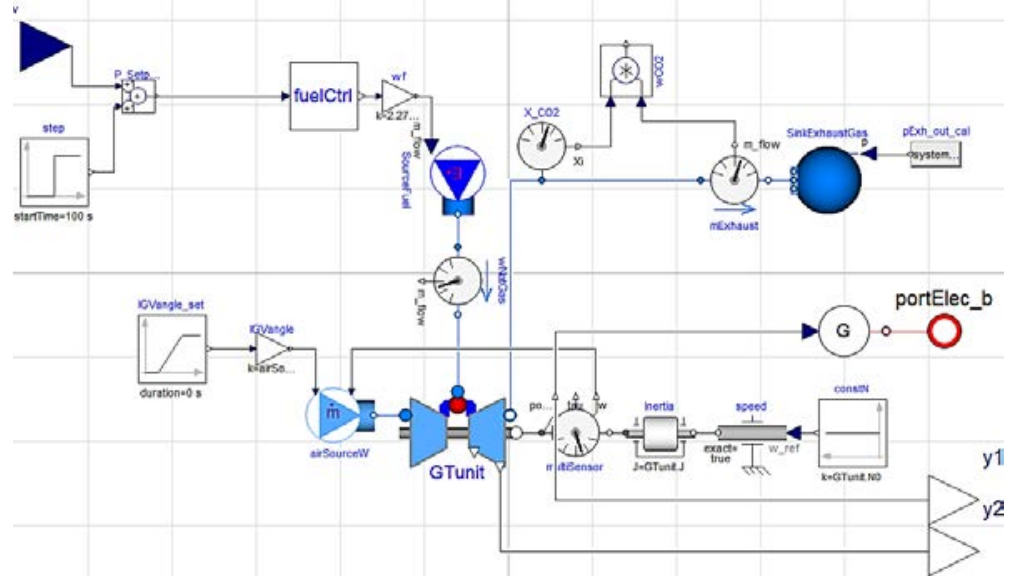

Figure 7-4. Detailed view of the reworked SES plant model.

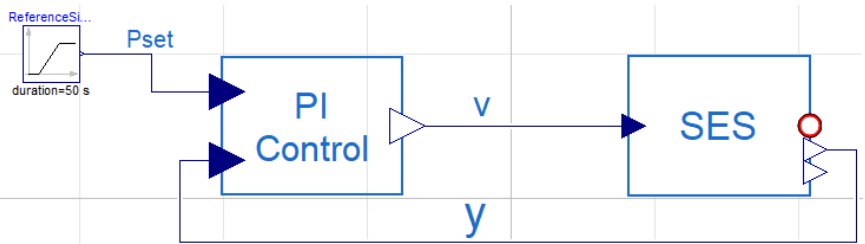

(a) 


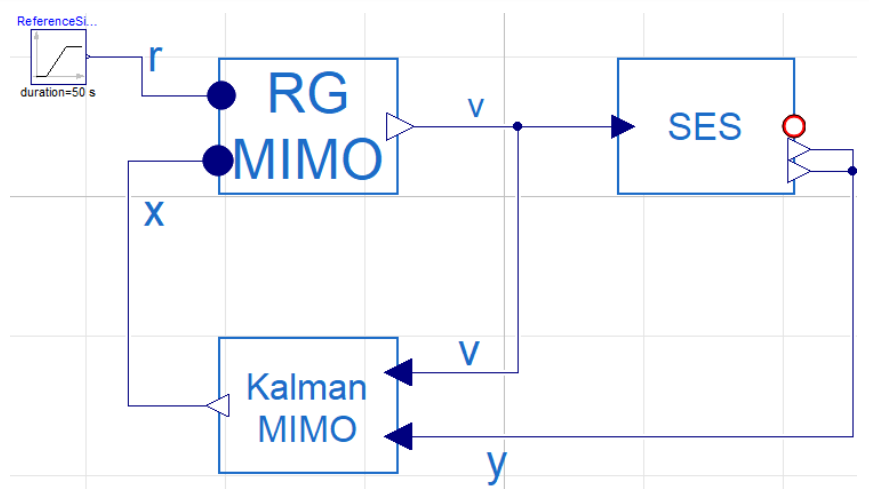

(b)

Figure 7-5. Tested control schemes.

To test the consistency of the "no-bus" interface with the plug-and-play philosophy, the reworked SES model was coupled with two different control systems. First, a traditional PID controller was implemented in a feedback loop with the SES model (Figure 7-5a). Then, a RGMIMO control scheme and a Kalman filter were implemented (Figure 7-5b). In the former configuration, the controller only needs the value of the controlled variable $\left(y_{1}\right)$. In the latter configuration, the Kalman filter needs to monitor both the output variables $\left(y_{1}\right.$ and $\left.y_{2}\right)$ to derive the state variable estimate that will be provided to the RG-MIMO.

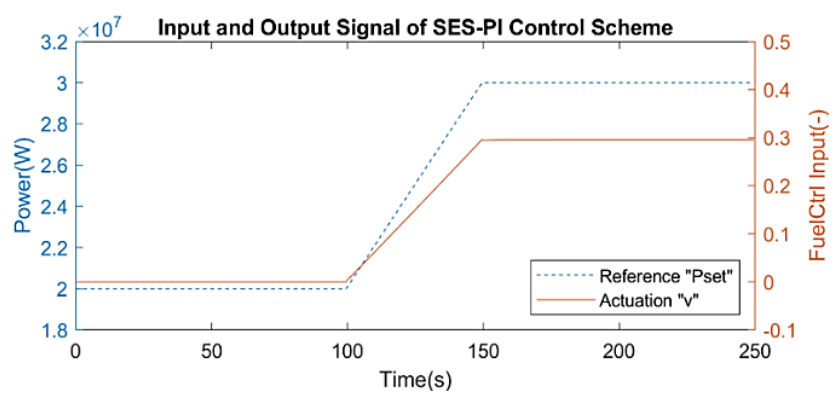

(a)

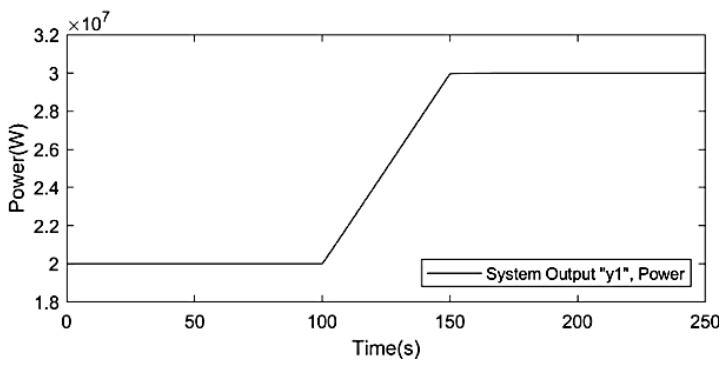

(b)

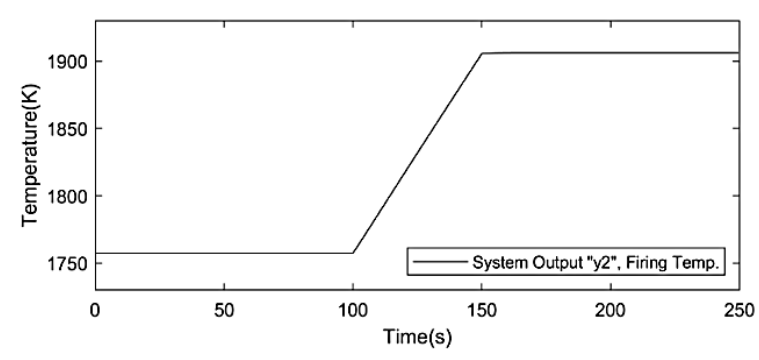

(c)

Figure 7-6. Simulation outcomes of the PID-based control scheme. 


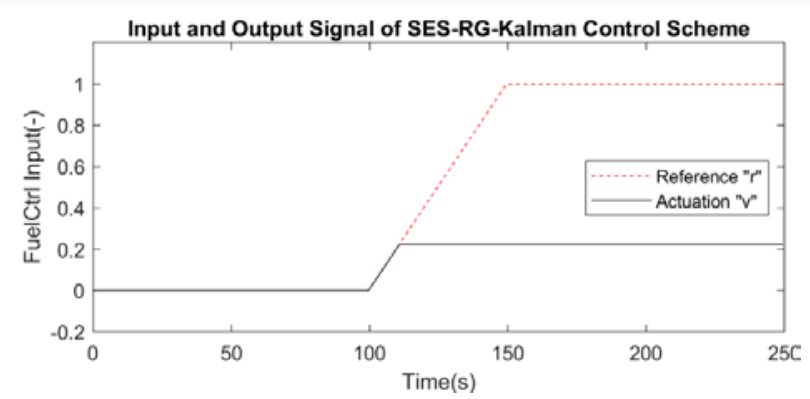

(a)

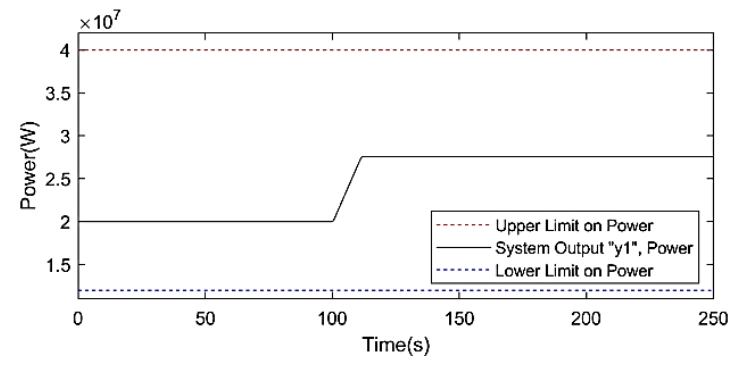

(b)

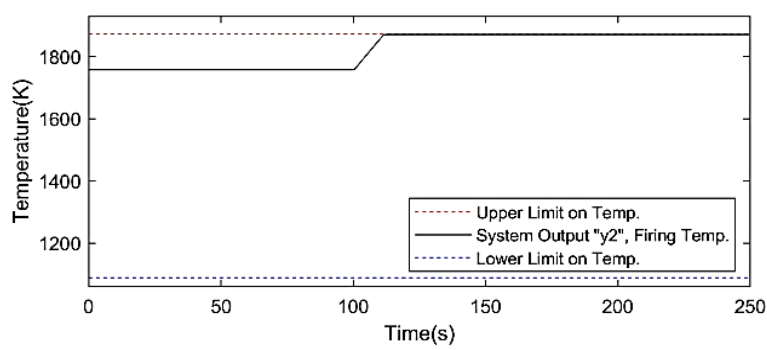

(c)

Figure 7-7. Simulation outcomes of the RG-Kalman-based control scheme.

Two control schemes requiring different output variables to derive the control action were tested but the plant model was not modified. In case additional output variables were needed by the controller, it would be sufficient to implement additional output interface to make them available. To prove the performance of the developed control schemes, a couple of operational transients were simulated. In Figure 7-5 and in Figure 7-6, the simulation outcomes of the PIDbased scheme and the RG-based scheme are shown, respectively. The former set of plots show the good set-point tracking capabilities ensured by the PID controller, and the evolution of the output variables. The latter set of plots show the constraint-verification capabilities ensured by the RG scheme.

\section{CONCLUSIONS}

In the first part of this report, a RG-based scheme that allows accounting for implicit constraints without increasing the size of the power dispatch optimization problem was presented. In current practice, the RG algorithm is a scheme placed between the power dispatcher and the PID controllers to enforce state and control constraints by modifying the set-point trajectories. The proposed configuration foresees an iterative loop between the power dispatcher and the RG to account for both the explicit constraints and the implicit constraints. To provide the RG with the needed state-space representation matrices, a data-driven procedure that allows reconstructing the state of the system and deriving a LTI system model was developed. In particular, State Selection and System Identification algorithms were built and integrated into the iterative loop. Different schemes characterized by increasing level of complexity were proposed. To assess the performance of the developed scheme, a two-unit test case was developed, and the obtained 
simulation outcomes were shown.

In the second part of the report, the preliminary version of the new Modelica library of control system elements was presented. Then, a new plug-and-lay paradigm was proposed for the configuration of the plant models. Some aspects of the current configuration of the IES unit components, e.g., the encapsulation of the control schemes into dedicated blocks, are consistent with the "plug-and-play" philosophy. Other features, e.g., the system buses collecting the input and the output variables, are not. For this reason, once listed and described the limits of the current configuration, necessary modifications to the plant model interface were presented. As a test-case, the SES model in the RAVEN-based HYBRID framework was reworked, i.e., two different control schemes were applied to the same plant model. 


\section{NOMENCLATURE}

Roman Symbols

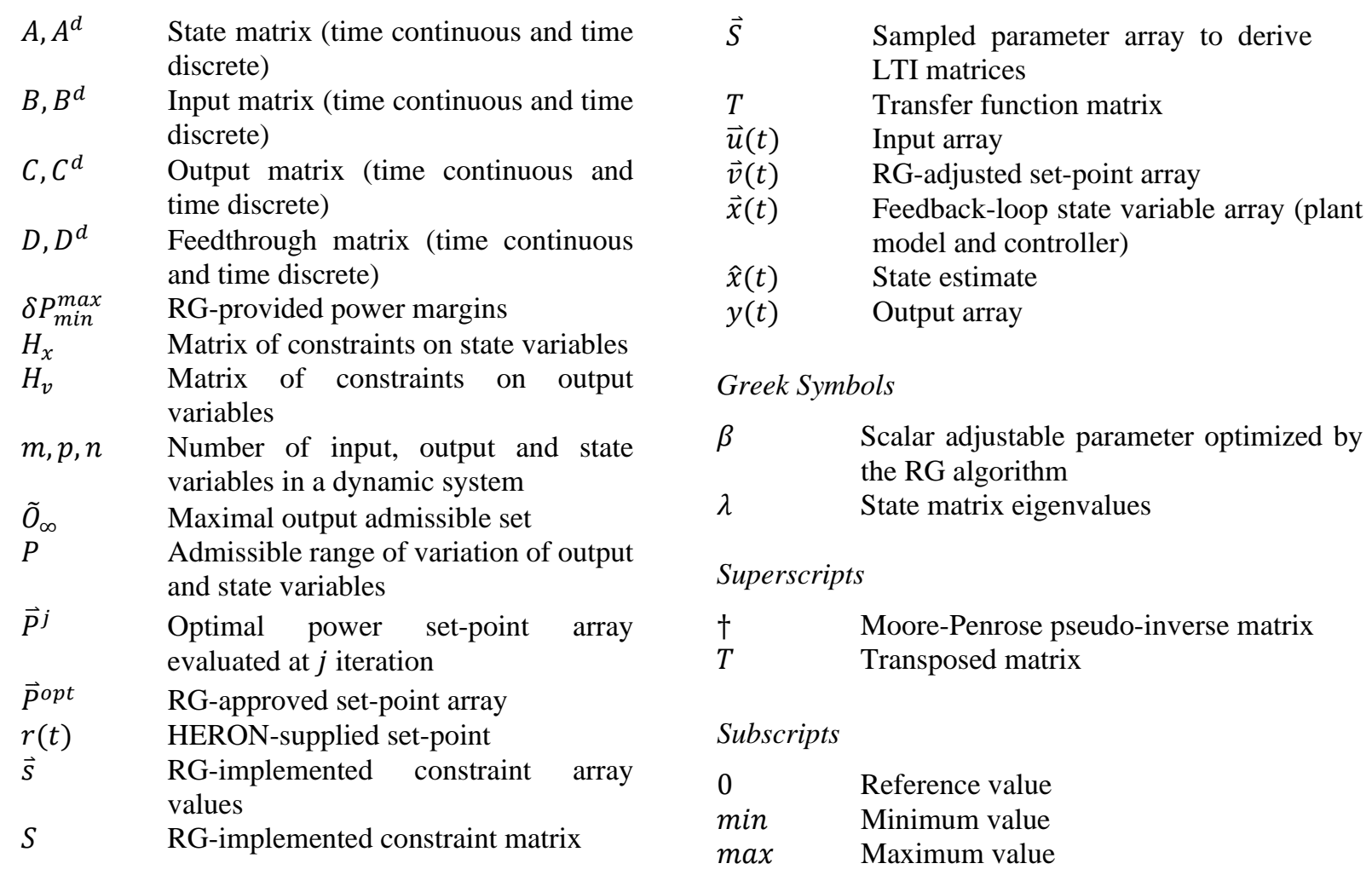

\section{REFERENCE}

[1] P.W. Talbot, A. Gairola, P. Prateek, A. Alfonsi, C. Rabiti, R.D. Boardman, "HERON as a Tool for LWR Market Interaction in a Deregulated Market”. INL/EXT-19-56933 (2020).

[2] D. Kourounis and O. Schenk, "Constraint handling for gradient-based optimization of compositional reservoir flow”, Computational Geosciences, 19, 1109-1122 (2015).

[3] E. Garone, S. Di Cairano, I. Kolmanovsky, "Reference and command governors for systems with constraints: A survey on theory and applications,” Automatica, 75, 306-328 (2017).

[4] A. Bemporad and E. Mosca, "Constraint fulfilment in Control Systems via predictive reference management," Proceedings of the $33^{\text {rd }}$ Conference on Decision and Control, 3017-3022, Lake Buena Vista, FL (1994).

[5] I. V. Kolmanovsky, E. Garrone, S. Di Cairano, "Reference and command governors: a tutorial on their theory and automotive applications,” Proceedings of 2014 ACC, Portland (OR), June 4-6 (2014).

[6] D. Rowell, “State-space representation of LTI systems”, MIT, Cambridge, (2002). Retrieved from http://web.mit.edu/214/www/Handouts/StateSpace.pdf. Accessed July 28 (2015).

[7] C. Rabiti, A. Alfonsi, J. Cogliati, D. Mandelli, R. Kinoshita, S. Sen, C. Wang, J. Chen, "RAVEN User Manual”, INL/EXT-15-34123 (2015).

[8] A. Alfonsi, C. Rabiti, D. Mandelli, J. Cogliati, C. Wang, P.W. Talbot, D.P. Maljovec, C. Smith, "RAVEN Theory Manual”, INL/EXT-16-38178 (2016).

[9] A. Alfonsi, C. Rabiti, D. Mandelli, J. Cogliati, C. Wang, P.W. Talbot, D.P. Maljovec, C. Smith, "RAVEN User Guide”, INL/EXT-18-44465 (2018).

[10] I. Guyon, J. Weston, S. Barnhill, V. Vapnik, “Gene selection for cancer classification using support vector machines”, Machine Learning, 46, 389-422 (2002). 
[11] J.L. Proctor, S.L. Brunton, J.N. Kutz, "Dynamic mode decomposition with control”, SIAM Journal on Applied Dynamical Systems, 15(1), 142-161 (2016).

[12] P.J. Schmid, "Dynamic mode decomposition of numerical and experimental data", Journal of Fluid Mechanics, 656(1), 5-28 (2010).

[13] E.H. Moore, "On the reciprocal of the general algebraic matrix", Bulletin of the American Mathematical Society, 26, 394-395 (1920).

[14] A. Casavola, E. Garone, F. Tedesco, "Distributed reference management strategies for a networked water distribution system”, 18 ${ }^{\text {th }}$ IFAC World Congress, Milan (Italy), August 28 - September 2 (2011).

[15] F. Tedesco, A. Casavola, E. Garone, "A distributed parallel command governor strategy for the coordination of multi-agent networked systems", $4^{\text {th }}$ IFAC Nonlinear Model Predictive Control conference, Noordwijkerhout, NL, August 23-27 (2012).

[16] R. Ponciroli, G. Maronati, R.B. Vilim, "Development of a Reference Governor-based Control Scheme for Integrated Energy Systems”, ANL/NSE-19/17 (2019).

[17] United States Nuclear Regulatory Commission (USNRC), “GE BWR/4 Advanced Technology Manual”, General Electric (2011).

[18] M.M. Tiller, "Introduction to Physical Modeling with Modelica”, Kluwer Academic Publishers (2001).

[19] J.M. Maciejowski, "Predictive control with constraints", Prentice Hall, London (2002).

[20] R. Ponciroli, S. Passerini, R.B. Vilim, "Innovative Control Strategy for Fast Runback Operational Transient Applied to SMRs”, Nuclear Technology, 191(2), 151-166 (2015). 


\section{Argonne}

\section{Nuclear Science and Engineering (NSE) Division}

Argonne National Laboratory

9700 South Cass Avenue, Bldg. 208

Argonne, IL 60439

www.anl.gov

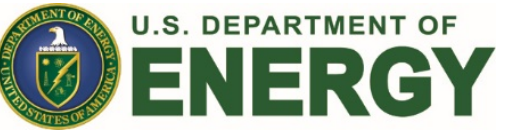

Argonne National Laboratory is a U.S. Department of Energy laboratory managed by UChicago Argonne, LLC 